DRAFT VERSION MAY 7, 2018

Preprint typeset using LATEX $_{\mathrm{E}}$ style emulateapj v. 11/26/04

\title{
THE SL2S GALAXY-SCALE LENS SAMPLE. II. COSMIC EVOLUTION OF DARK AND LUMINOUS MASS IN EARLY-TYPE GALAXIES
}

\author{
Andrea J. RufF ${ }^{1,2 *}$, Raphä̈l Gavazzi ${ }^{3}$, Philip J. Marshall ${ }^{1,4}$, Tommaso Treu ${ }^{1 \dagger}$, Matthew W. Auger ${ }^{1}$, and \\ FLORENCE BRAUlT ${ }^{3}$ \\ Draft version May 7, 2018
}

\begin{abstract}
We present a joint gravitational lensing and stellar-dynamical analysis of 11 early-type galaxies (median deflector redshift $z_{\mathrm{d}}=0.5$ ) from Strong Lenses in the Legacy Survey (SL2S). Using newly measured redshifts and stellar velocity dispersions from Keck spectroscopy with lens models from Paper I, we derive the total mass density slope inside the Einstein radius for each of the 11 lenses. The average total density slope is found to be $\left\langle\gamma^{\prime}\right\rangle=2.16_{-0.09}^{+0.09}\left(\rho_{\text {tot }} \propto r^{-\gamma^{\prime}}\right)$, with an intrinsic scatter of $0.25_{-0.07}^{+0.10}$. We also determine the dark matter fraction for each lens within half the effective radius, $R_{\text {eff }} / 2$ and find the average projected dark matter mass fraction to be $0.42_{-0.08}^{+0.08}$ with a scatter of $0.20_{-0.07}^{+0.09}$ for a Salpeter IMF. By combining the SL2S results with those from the Sloan Lens ACS Survey (median $z_{\mathrm{d}}=0.2$ ) and the Lenses Structure and Dynamics survey (median $z_{\mathrm{d}}=0.8$ ), we investigate cosmic evolution of $\gamma^{\prime}$ and find a mild trend $\partial\left\langle\gamma^{\prime}\right\rangle / \partial z_{\mathrm{d}}=-0.25_{-0.12}^{+0.10}$. This suggests that the total density profile of massive galaxies has become slightly steeper over cosmic time. If this result is confirmed by larger samples, it would indicate that dissipative processes played some role in the growth of massive galaxies since $z \sim 1$.
\end{abstract}

Subject headings: galaxies: fundamental parameters — gravitational lensing —

\section{INTRODUCTION}

Early-type (i.e. elliptical and lenticular) galaxies in the local universe are considered to be simple objects (e.g. Bertin \& Stiavelli 1993; Merritt 1999; Ciotti 2009). In the central few kpc most of their mass is dominated by stars, while at larger radii there is convincing evidence at least for the most massive systems - that dark matter halos are dominant. Their stellar populations are relatively simple, dominated by old stars with little or negligible star formation (e.g. Renzini 2006). Remarkably, many global properties, ranging from the chemical composition of their stars to their size, luminosity and mass of the central black hole, correlate tightly with their stellar velocity dispersion, $\sigma$ (e.g. Bernardi et al. 2005; Graves et al. 2009).

In spite of this suspected simplicity, their formation and evolution are still poorly understood, and therefore they are the subject of many observational and theoretical investigations. A number of observational facts have proved difficult to explain by theoretical models. These include: i) the tightness of the empirical correlations with $\sigma$ (e.g., Bernardi et al. 2005; Graves et al. 2009; Nipoti et al. 2009a); ii) the so-called downsizing trend of their stellar populations, i.e. the correlation between mean stellar age and present day stellar mass (e.g. Thomas et al. 2005; Treu et al. 2005; van der Wel et al. 2005; Juneau et al. 2005; di Serego Alighieri et al. 2005); iii) the evolution of the upper end of their mass function

\footnotetext{
${ }^{1}$ Physics department, University of California, Santa Barbara, CA 93106, USA

${ }^{2}$ University of Melbourne, Parkville 3010, Victoria, Australia

${ }^{3}$ Institut d'Astrophysique de Paris, UMR7095 CNRS - Université Pierre et Marie Curie, 98bis bd Arago, 75014 Paris, France

${ }^{4}$ KIPAC, P.O. Box 20450, MS29, Stanford, CA 94309, USA

* aruff@unimelb.edu.au

$\dagger$ Packard Research Fellow
}

since $z \sim 1$ (e.g. , Bundy et al. 2005, 2007; Cimatti et al. 2006; van der Wel et al. 2009; Hopkins et al. 2010); iv) the unusually compact size of high redshift massive red galaxies (e.g., Treu et al. 1998; Daddi et al. 2005; van Dokkum et al. 2008; Saracco et al. 2009; Cassata et al. 2010; Mancini et al. 2010; Newman et al. 2010). From a theoretical standpoint, it is clear that understanding the interplay between baryons, black holes, and dark matter is essential to develop a scenario that can quantitatively reproduce all observations. The physical processes that need to be accurately modeled in a successful theory appear to include black hole accretion and the related energy and momentum feedback (e.g., Croton et al. 2006; Ciotti et al. 2009), dry and wet major mergers (e.g., Khochfar \& Silk 2006; Ciotti et al. 2007; Robertson et al. 2006), as well as minor mergers and dry accretion of minor satellites (e.g., Naab et al. 2009; Hopkins et al. 2010).

Most of the observational studies, including those listed in the previous paragraph, are concerned with global parameters of early-type galaxies. An entirely new line of investigation can be opened up if we are able to dissect early-type galaxies and map their internal dynamical structure as a function of cosmic time. By decomposing the internal mass distribution of early-type galaxies into luminous and dark components we can start addressing the following questions, the answers to which would provide essential clues as to their formation and evolution. How and when is mass assembled to form earlytype galaxies? How are baryons converted into stars and accumulated inside dark matter halos? Is the mass density profile of early-type galaxies comparable to that observed in numerical simulations? Do isolated early-type galaxies undergo internal structural and dynamical evolution? As numerical simulations of early-type galaxies become more and more realistic, detailed knowledge of their internal structure (e.g. their distribution functions) 
will provide more and more stringent tests of the current paradigm of structure formation (e.g. Meza et al. 2003; Naab et al. 2007; Oñorbe et al. 2007; Lackner \& Ostriker 2010).

Great progress in answering these questions has been achieved in the past few years with the systematic study of early-type galaxies acting as strong gravitational lenses. For these systems, strong lensing provides an absolutely calibrated measurement of mass at a fiducial radius (the Einstein radius), which is typically comparable in size to the effective radius. By combining this mass tracer with traditional diagnostics such as stellar velocity dispersion (Miralda-Escude 1995; Natarajan \& Kneib 1996; Treu \& Koopmans 2002), and stellar mass maps from multicolor imaging and/or spectroscopy, one can break many of the degeneracies inherent to each method alone, including the mass-anisotropy degeneracy, bulge-halo degeneracy, and the stellar mass/initial mass function (IMF) degeneracy (e.g. Koopmans \& Treu 2003; Treu \& Koopmans 2004; Treu et al. 2010). Additional information can be gathered with the addition of weak-lensing (Gavazzi et al. 2007; Jiang \& Kochanek 2007; Lagattuta et al. 2010), although at the moment this is not possible for individual galaxies. The SLACS team applied this methodology to a sample of more than 80 early-type galaxies (Bolton et al. 2006, 2008a; Auger et al. 2009), which have been shown to be indistinguishable from equally massive non-lensing early-type galaxies in terms of their internal properties and environment (Bolton et al. 2006; Treu et al. 2006, 2009).

Among the most relevant findings of SLACS is that the total mass-density profile $\rho_{\text {tot }} \propto r^{-\gamma^{\prime}}$ of early-type galaxies is close to isothermal with $\gamma^{\prime}=2.085_{-0.018}^{+0.025}$ with intrinsic scatter less than 0.1 (Koopmans et al. 2009; Barnabè et al. 2009; Auger et al. 2010), even though neither the stars nor the dark matter obey a simple power law profile (see also Wucknitz et al. 2004). This "bulge-halo conspiracy", similar to the disk-halo conspiracy found for spiral galaxies (e.g. van Albada \& Sancisi 1986), has implications both for lensing studies but also for galaxy formation studies. Since the total mass density profile is preserved by dry-mergers (Dehnen 2005; Kazantzidis et al. 2006; Nipoti et al. 2009b), and dark matter-only profiles are not isothermal (e.g. Navarro et al. 2010), the isothermal nature has to be established through dissipational processes (e.g., Koopmans et al. 2006). The other main finding of the SLACS survey is that (assuming a constant stellar IMF) the fraction of dark matter $f_{\mathrm{DM}}$ within a fixed fraction of the effective radius increases with galaxy mass or stellar velocity dispersion (see also Jiang \& Kochanek 2007; Grillo et al. 2008; Cardone et al. 2009; Cardone \& Tortora 2010). Possible explanations for this trend are: varying efficiency in converting baryons into stars as a function of halo mass, varying inner slope of the dark matter halo with mass, and evolutionary processes. For example, dry mergers can increase the fraction of dark matter within the effective radius (e.g., Nipoti et al. 2009b).

The SLACS, however, sample is limited to low redshift by the selection function of the parent sample of SDSS luminous galaxies. Therefore, evolutionary studies with the SLACS sample are limited to a short baseline. Suitable samples of strong lenses are smaller at high redshift. In fact, most of the strong lensing galaxies known to date at $z>0.4$ are too faint and/or dominated by strongly lensed bright quasars to allow for detailed kinematic studies of the deflector. Of the handful of exceptions (e.g. , Faure et al. 2008; Lagattuta et al. 2010), only a few of them have published lensing and dynamical analysis (Treu \& Koopmans 2002; Ohyama et al. 2002; Treu \& Koopmans 2004; Suyu et al. 2010). These early studies found results similar to SLACS, i.e. the internal slope $\gamma^{\prime}$ is close to isothermal, and the central dark matter fraction appears to increase with mass as found by the LSD Survey (Treu \& Koopmans 2004). However, the results also hint that things may have been different at $z \sim 1$ when the universe was less than half its present age: the scatter in $\gamma^{\prime}$ might have been larger (Treu \& Koopmans 2004) and perhaps even the average might have been different (Koopmans et al. 2006). Unfortunately, current samples beyond $z \sim 0.4$ with lensing and dynamical data are too small to probe the evolution of the internal structure of early-type galaxies, its scatter and trends with mass at the same time.

In this paper we present the first detailed study of a sample of early-type lens galaxies identified by the Strong Lenses in the Legacy survey (SL2S). We use new spectroscopic data obtained at the W.M. Keck telescopes, in combination with multicolor photometry from the CFHT Legacy Survey to infer dynamical and stellar mass for the deflector galaxies. This imaging data-set is complemented by HST imaging that allowed to confirm the lensing nature of the SL2S candidates and detailed lens modeling. The newly measured source and deflector redshifts are combined with gravitational lens models from Paper I (Gavazzi et al., in preparation) to infer lensing masses. We determine the total mass density profile of the earlytype lens galaxies (quantified by $\gamma^{\prime}$ ) and their central dark matter fraction $\left(f_{\mathrm{DM}}\right)$. The median redshift of the sample is $z=0.494$, providing an ideal complement to the earlier SLACS and LSD samples. By combining the three samples we investigate evolutionary trends in these quantities.

This paper is organized as follows. In Section 2 we describe the SL2S survey, and how the galaxy-scale lens candidates were selected. We then present our spectroscopic measurements in Section 3 before combining them with lens model parameters to investigate the mass structure of massive galaxies since redshift 0.9 in Section 7 . In Section 4 we incorporate stellar mass estimates in order to separate the dark and luminous components of the lens galaxies. The cosmic evolution of the total mass density slope and dark matter fraction are discussed in Section 8. After a brief discussion of our results in Section 9 we conclude in Section 10. Throughout this paper magnitudes are given in the AB system. We assume a concordance cosmology with matter and dark energy density $\Omega_{m}=0.3, \Omega_{\Lambda}=0.7$, and Hubble constant $\mathrm{H}_{0}=70$ $\mathrm{km} \mathrm{s}^{-1} \mathrm{Mpc}^{-1}$.

\section{STRONG LENSES IN THE LEGACY SURVEY: SL2S}

In this section we describe our feeder survey, the CFHT Legacy Survey, and how we identified our new sample of galaxy scale lenses at intermediate redshift. A more detailed description is given in Paper I.

Strong lensing candidates were selected from the Canada-France-Hawaii Telescope Legacy Survey 
$(\text { CFHTLS })^{7}$. In brief, the survey consists of two main components of sufficient depth and image quality to be interesting for lens searching ${ }^{8}$. Both are imaged in the $u^{*}, g, r, i$ and $z$ bands with the $1 \operatorname{deg}^{2}$ field-of-view Megacam Camera. The multi epoch Deep survey covers 4 pointing of $1 \mathrm{deg}^{2}$ each. Two different image stacks were produced: D- 85 contains the $85 \%$ best seeing images whereas the D- 25 only includes the $25 \%$ best seeing images. For finding lenses we only considered these latter, better resolution stacks. They reach a typical depth of $u^{*} \simeq 26.18, g=25.96 \simeq 25.47, r \simeq 25.43$, $i \simeq 25.08$ and $z \simeq 24.57$ (80\% completeness for point sources) with typical FWHM point spread functions of $00^{\prime \prime} 75,0^{\prime \prime} 69,0$, $64,00^{\prime \prime} 62$ and $0{ }^{\prime \prime} 61$, respectively. The Wide survey is a single epoch imaging survey, covering some $171 \mathrm{deg}^{2}$ in 4 patches of the sky. It reaches a typical depth of $u^{*} \simeq 25.35, g \simeq 25.47, r \simeq 24.83, i \simeq 24.48$ and $z \simeq 23.60$ (AB mag of $80 \%$ completeness limit for point sources) with typical FWHM point spread functions of

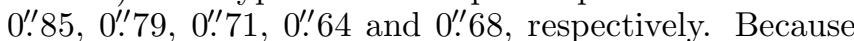
of the greater area, the Wide component is our main provider of lens candidates.

Images from both the Deep and Wide survey modes were analyzed to find strong lens candidates using several algorithms, as described by Cabanac et al. (2007) at the group and cluster mass scales, and by Gavazzi et al. (in preparation) at the galaxy scale with the RingFinder algorithm. The ring-detecting algorithm is aimed at detecting compact rings around centers of isolated galaxies $\left(<10^{13} h^{-1} M_{\odot}\right)$, and works by focusing on the achromatic image excesses around early-type lens galaxies that are indicative of the presence of lensed arcs. For each of a sample of pre-selected bright $\left(i_{\mathrm{AB}} \leq 22.5\right)$ red galaxies, a scaled, PSF-matched version of the $i$-band cutout image was subtracted from the $g$-band image of the same system. The rescaling in this operation is performed such that the early-type galaxy light is efficiently removed, leaving only objects with an SED different from that of the target galaxy. These (typically) blue residuals are then characterized with an object detector, and analyzed for their position, ellipticity, and orientation, and those showing characteristic properties of lensed arcs are kept as lens candidates. A sample of several hundred good candidates were visually inspected, and ranked for followup with $H S T$.

Currently, 65 CFHT galaxy-scale lens candidates have been observed firstly with ACS, then WFPC2, and finally with WFC3 as snapshot programs over cycles 15 , 16 and the ongoing cycle 17. Details of these observations are given in Paper I. Approximately $50 \%$ of the lens candidates were confirmed as lenses in this way. The sources are all faint blue galaxies, with very few showing signs of an active nucleus. Those with the most convincing lens models (see Paper I) were selected for spectroscopic follow up to obtain high precision redshifts for lens and source galaxies, and lens galaxy velocity dispersions. These observations are described in the next section.

\section{SPECTROSCOPIC OBSERVATIONS}

Follow up spectroscopy of 17 selected lens candidates was obtained using the Low Resolution Imaging Spectro-

\footnotetext{
7 See http://www.cfht.hawaii.edu/Science/CFHLS/ and links therein for a comprehensive description

8 http://terapix.iap.fr/rubrique.php?id_rubrique=259
}

TABLE 1

OBSERVING LOGS

\begin{tabular}{ccccccc}
\hline \hline run & obsdate & graname & grisname & slit & time & seeing \\
& & & & & & \\
\hline 1 & 20 Jul 2006 & $831 / 8200$ & $600 / 4000$ & $1^{\prime \prime} 5$ & 20 & $0^{\prime \prime} 84$ \\
2a & 23 Dec 2006 & $831 / 8200$ & $300 / 5000$ & $1^{\prime \prime} 5$ & 60 & $0^{\prime \prime} 60$ \\
2b & 23 Dec 2006 & $831 / 8200$ & $300 / 5000$ & $1^{\prime \prime} 5$ & 40 & $0^{\prime \prime} 80$ \\
3a & 13 Sep 2007 & $400 / 8500$ & $400 / 3400$ & $1^{\prime \prime} 0$ & 45 & $0^{\prime \prime} 73$ \\
3b & 13 Sep 2007 & $400 / 8500$ & $400 / 3400$ & $1^{\prime \prime} 0$ & 30 & $0^{\prime \prime} 75$ \\
4a & 14 Sep 2007 & $400 / 8500$ & $400 / 3400$ & $1^{\prime \prime} 0$ & 30 & $0^{\prime \prime} 55$ \\
4b & 14 Sep 2007 & $600 / 7500$ & $600 / 4000$ & $0^{\prime \prime} 7$ & 30 & $0^{\prime \prime} 55$ \\
4c & 14 Sep 2007 & $831 / 8200$ & $600 / 4000$ & $1^{\prime \prime} 0$ & 90 & $0^{\prime \prime} 55$ \\
5 & 9 Sep 2009 & $600 / 7500$ & $300 / 5000$ & $1^{\prime \prime} 0$ & 30 & $1^{\prime \prime \prime} 0$ \\
6 & 14 Jan 2010 & $600 / 7500$ & $300 / 5000$ & $1^{\prime \prime} 0$ & 45 & $1^{\prime \prime} 3$ \\
& & & & & & \\
\hline
\end{tabular}

Note. - The exposure time is given in minutes. The plate scale for both the red and blue chips is $0.135 /$ pixel for observations from 2009 and after. For observing runs before 2009 , the red chip had plate scale of $0^{\prime \prime} 211 /$ pixel.

graph (LRIS) on the Keck I telescope over six nights from 2006 to 2010 , with the aim of measuring the deflector and source redshifts and the velocity dispersion of the deflector. Because of incompleteness in the execution of the $H S T$ snapshot observations mentioned above, we have spectra of 6 systems where $H S T$ data is not yet available. These lens candidates, however, were very promising on the basis of their CFHT images alone. In this section we describe the observations and their analysis in some detail, outlining our methodology for measuring the lens redshifts, source redshifts, and lens velocity dispersions.

\subsection{Details of the observations and data reduction}

Observations were made in long slit mode, with the slit centered on the deflector. The slit orientation was chosen to maximize both the source flux and the spatial separation of the deflector and arc. The total flux is dominated by the deflector, so maximizing the source flux maximizes the likelihood of measuring the source redshift, $z_{\mathrm{s}}$. Spatial separation within the slit also separates the deflector and arc traces in the 2D spectra, allowing us to look for source emission lines directly, as discussed in Section 3.4. The seeing ranged between $0 . " 55$ and 1". 3 and total exposure times varied from 20 to $45 \mathrm{~min}$, typically with two consecutive 15 min intervals. Between each exposure, we dithered along the slit by $10^{\prime \prime}$ to improve defect removal and sky subtraction. A summary of each observing run is given in Table 1.

The later setups took greater advantage of the blue sensitivity of LRIS. We found that the most effective method to measure all three quantities was using a 680 dichroic, so that the deflector redshift and velocity dispersion could be measured in the blue. The 680 dichroic allowed us to choose a large wavelength coverage for the red, which was useful in detecting $[\mathrm{O}$ II] out to $z>1.1$.

The data was reduced using a Python pipeline (developed by MWA). On the red side, the night sky lines were used to determine the wavelength solution, while standard arclamps were used on the blue side. Two different extraction windows were chosen: one wide extraction window to ensure that light from the source was also included in the final spectrum (half widths between 5 and 12 pixels from the central trace) and another narrower 

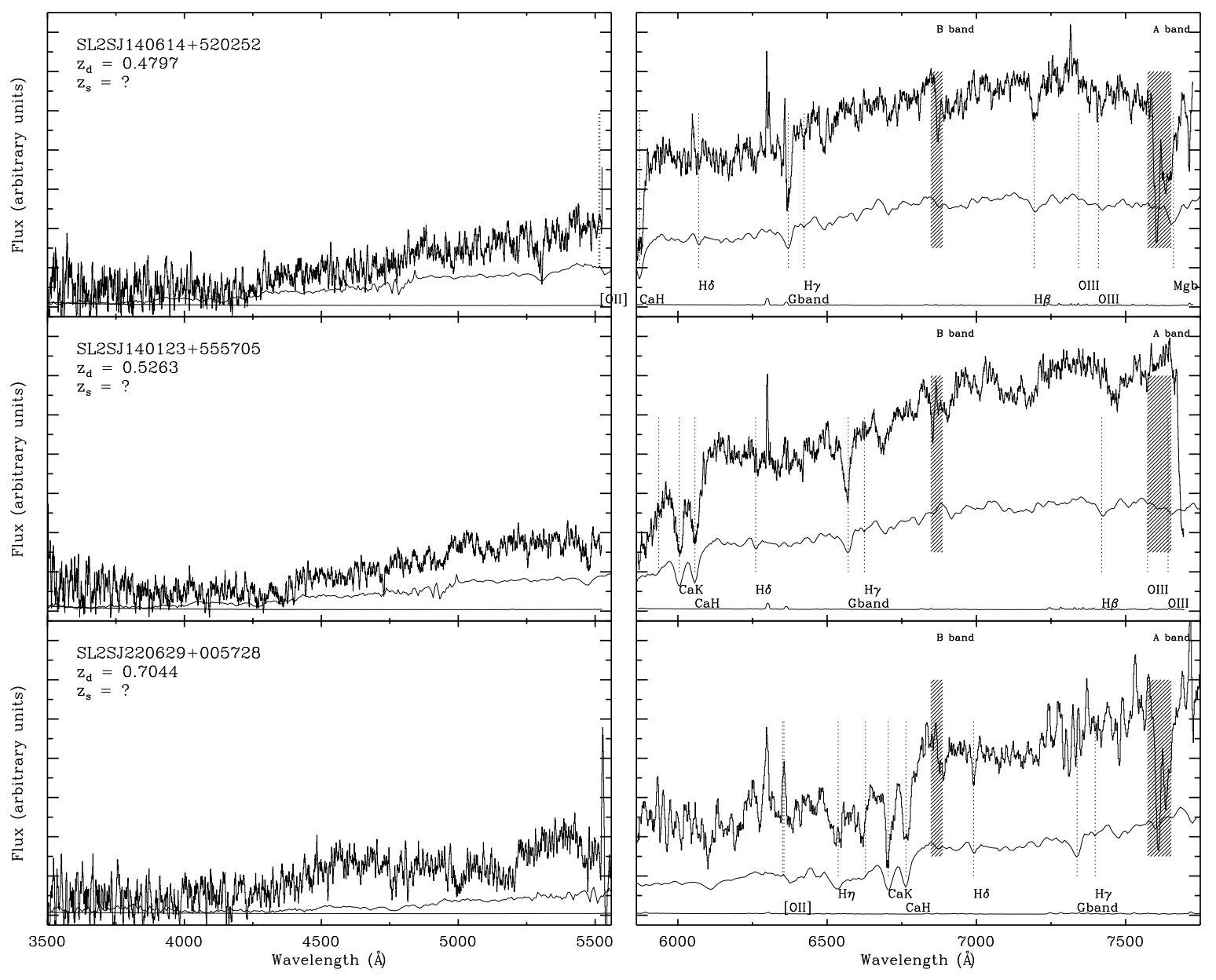

FIG. 1.- Keck/LRIS spectra for 17 SL2S lenses. An elliptical galaxy template is shown in a thin black line beneath each spectrum. The dotted black lines mark features of the lens galaxy, while source emission lines are highlighted in red. The shaded regions indicate A and B band telluric absorption features.

extraction window to increase the signal to noise ratio, which is important for measuring velocity dispersions. The reduced spectra are shown in Figure 1.

\subsection{Measuring deflector redshifts}

Deflector redshifts were measured using the centroids and known rest frame wavelengths of prominent absorption features. In the majority of cases, a minimum of the $\mathrm{Ca}$ II $\mathrm{H}$ and $\mathrm{K}$ lines were used, however other absorption features were also used when available. The additional absorption features used were:

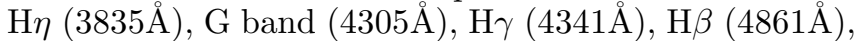

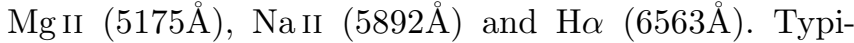
cally, between three and five absorption features were centroided to measure the redshift. The measured redshifts are listed in Table 2.

\subsection{Measuring deflector velocity dispersions}

Velocity dispersions were measured by fitting combinations of stellar spectra over regions with prominent absorption features and high signal to noise. Linear combinations of stellar spectra were used to fit a model to the data and calculate a velocity dispersion. A Python based implementation of the van der Marel (1994) velocity dispersion code, developed by MWA and described by Suyu et al. (2009), was used. We use a set of templates from the INDO-US stellar library containing spectra for a set of seven $\mathrm{K}$ and $\mathrm{G}$ giants with a variety of temperatures and spectra. K and G giants were used because they provide a good description of the spectra of our deflectors, as expected for massive ellipticals.

The value of the velocity dispersion, $\sigma$ for each deflector was determined by finding a consistent value over several spectral regions and features. If the mean signal to noise $(\mathrm{S} / \mathrm{N})$ ratio per rest-frame angstrom was $\gtrsim 10$

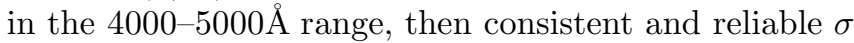
values could be measured. Of the 17 SL2S lenses, 12 spectra had sufficient $\mathrm{S} / \mathrm{N}$ to measure $\sigma$.

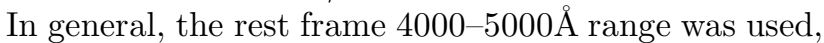
as the G-band absorption feature is often uncontaminated by atmospheric absorption, and contains no sharp changes in the continuum and the CCD efficiency is also good over this range. The average signal to noise ratio per angstrom in the $4000-5000 \AA$ range for the 12 objects with measured velocity dispersions is 24.8 .

Other regions that were used to fit the stellar templates

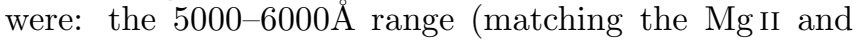
Na II absorption features) and the 3500-4000 $\mathrm{\AA}$ range was also used, but only where the depth and continuum fit to the spectra were good. Generally, three regions of the spectrum that produced good fits and consistent $\sigma$ values were used to calculate the final $\sigma$ and its associated 


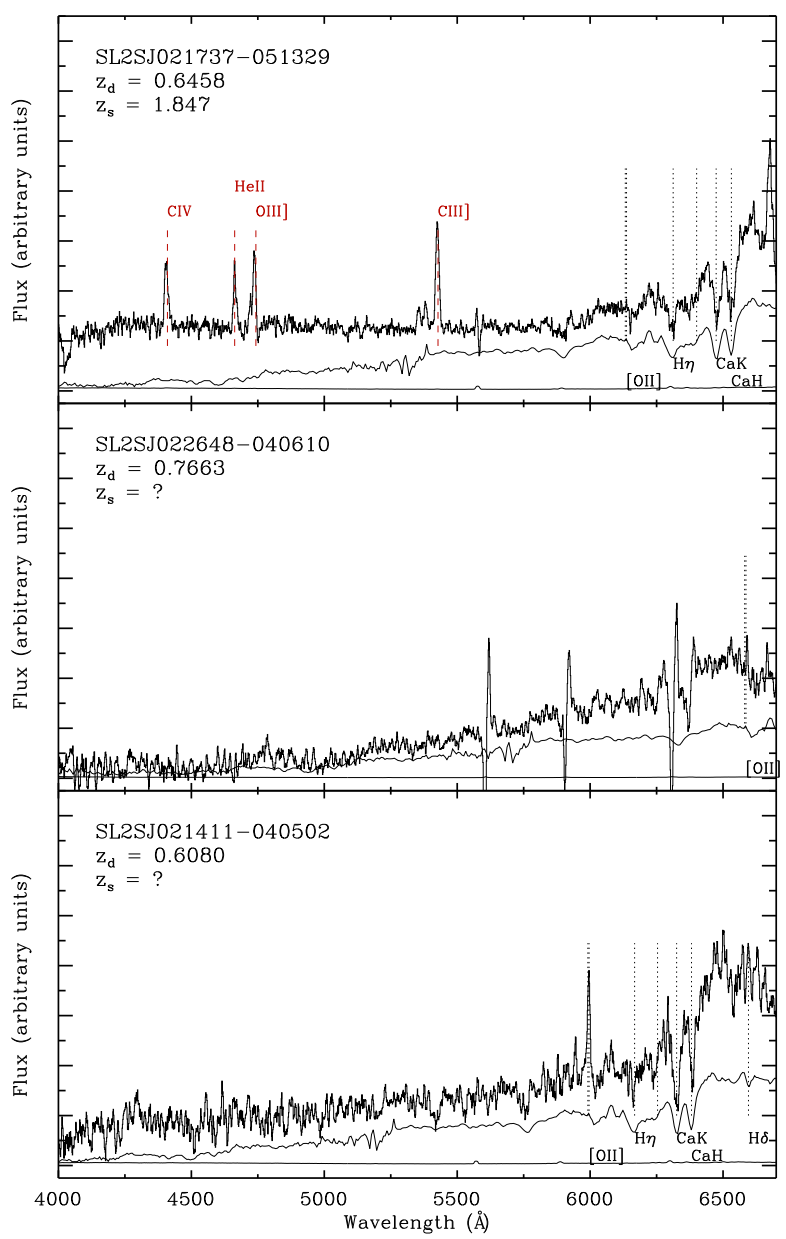

FIG. 1.- continued

uncertainty. For each lens, one of the models generated to measure $\sigma$ is shown in Figure 2. Regions where atmospheric absorption was a problem, or the templates did not produce a good fit were masked out, as shown in the grey regions of Figure 2. The results of the velocity dispersion measurements and the mean $\mathrm{S} / \mathrm{N}$ per angstrom in the rest frame $4000-5000 \AA$ range for each object are listed in Table 2. The mean measured $\sigma$ for the SL2S sample is $250 \mathrm{~km} \mathrm{~s}^{-1}$. The measured velocity dispersions were then corrected to a uniform physical aperture using the slit width, the size of the extraction window and the empirical power-law relation of Jørgensen et al. (1995). The corrected velocity dispersion, $\sigma_{e 2}$, measures the velocity dispersion at $R_{\text {eff }} / 2$, as used by Bolton et al. (2008b). Inside the effective radius, the relation is well described by a power law:

$$
\sigma_{\mathrm{e} 2}=\sigma_{\mathrm{ap}}\left(\frac{R_{\mathrm{eff}}}{2 r_{\mathrm{ap}}}\right)^{-0.04},
$$

where $2 r_{\text {ap }} \approx 2(x y / \pi)^{1 / 2}$ and where $x$ and $y$ are the width and length of the rectangular aperture.

\subsection{Measuring source redshifts}

Source redshifts were measured for five objects. Two objects, SL2SJ021737-051329 and SL2SJ141137+565119 $\left(z_{s}=1.847\right.$ and 1.420 , respectively $)$ have multiple source emission lines that were centroided to measure the redshift. As can be seen in Figure 1, SL2SJ021737-051329

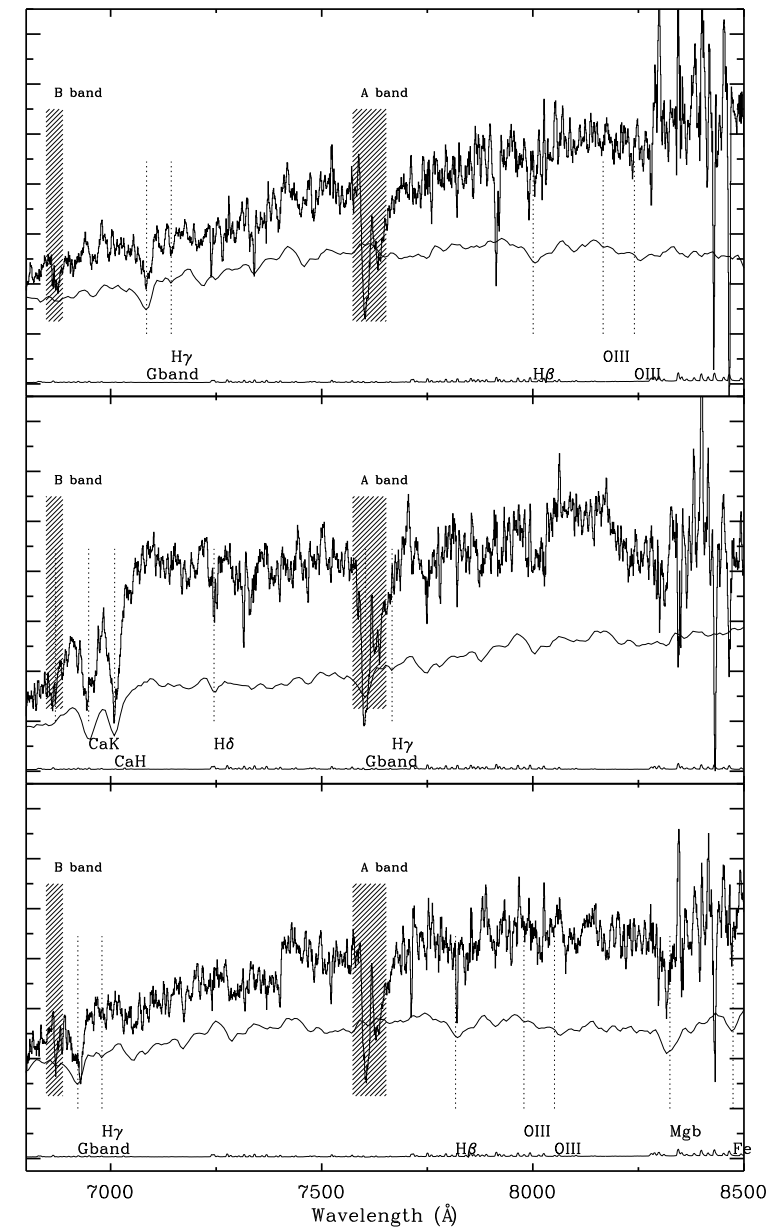

has narrow emission lines typical of Type II AGN (C IV $\lambda 1549$, He II $\lambda 1640$, O III] $\lambda 1666$ and C III] $\lambda 1909$ ). SL2SJ141137+565119 shows a clear splitting of the [O II] doublet as well as strong C III] and O III] emission.

The three remaining source redshifts were identified using [O II] $\lambda \lambda 3726.1,3728.8$ only. SL2SJ022610-042011 and SL2SJ022511-045433 $\left(z_{s}=1.232\right.$ and 1.1988, respectively) show a clear splitting of the doublet in the $2 \mathrm{D}$ spectra. For the objects where there was no clear splitting of the $[\mathrm{O} \mathrm{II}]$ doublet, the redshift measurement was more difficult. We used two methods to search for source emission lines. Firstly, we looked for emission lines in the $2 \mathrm{D}$ spectra at the expected position of the source trace. Secondly, we looked at the residuals from both a simple fit to a standard elliptical galaxy template and also the residuals from the velocity dispersion measurement, discussed in Section 3.3. Despite that three source redshifts were identified using [O II] alone, no other strong emission features are observed blue-ward of this feature, corroborating the identification, as we would expect to see other lines if the detected feature were redder (e.g. O III], $\mathrm{H} \alpha$ or $\mathrm{H} \beta$ ).

The 2D spectrum of SL2SJ022511-045433 shows a slight offset between the [O II] emission of the arc and the arclet. We estimate the difference in redshifts to be $\Delta z=0.0005$, corresponding to a relative motion of $\sim 150 \mathrm{~km} / \mathrm{s}$. This is most likely due to kinematic structure of the source. Consistent with this interpretation, 


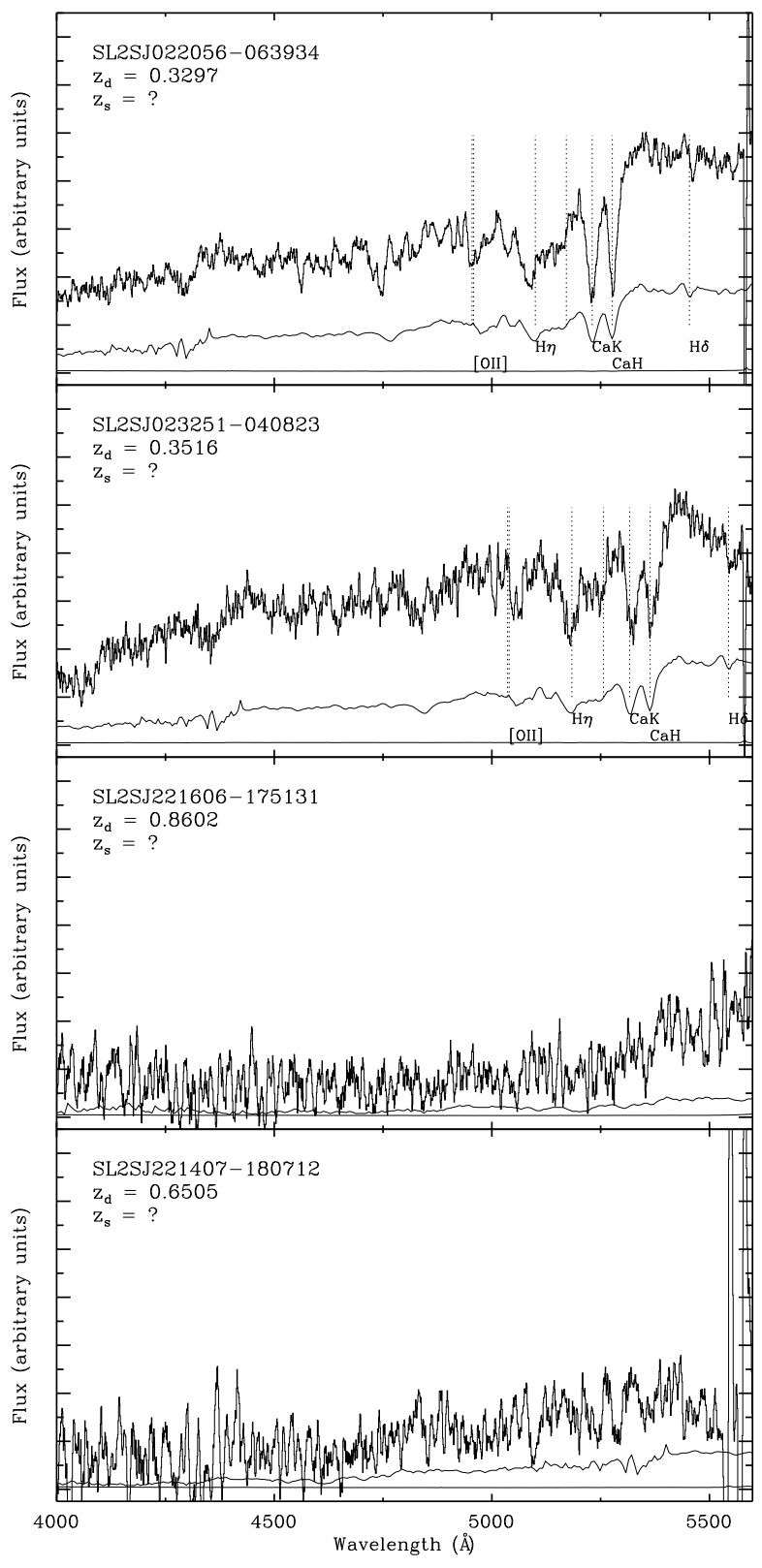

FIG. 1.- continued

the lens model indicates the presence of two separate peaks in the surface brightness distribution of the lens, i.e. possibly a pair of source galaxies (see Paper I for details).

\subsection{Systems with no source emission lines}

For the remaining 11 sources, for which no source emission lines could be detected, we must estimate the source redshift from whatever information we have. We first consider the the HST and CFHTLS photometry. We did not attempt to infer photometric redshifts, since the disentanglement of lens and source colors in the lowresolution, multi-filter CFHTLS data was deemed likely to lead to significant systematic uncertainty. Instead, we conservatively used the redshift distribution of the faint galaxies in the COSMOS survey to provide a broad probability density function (PDF) for $z_{\mathrm{s}}$.

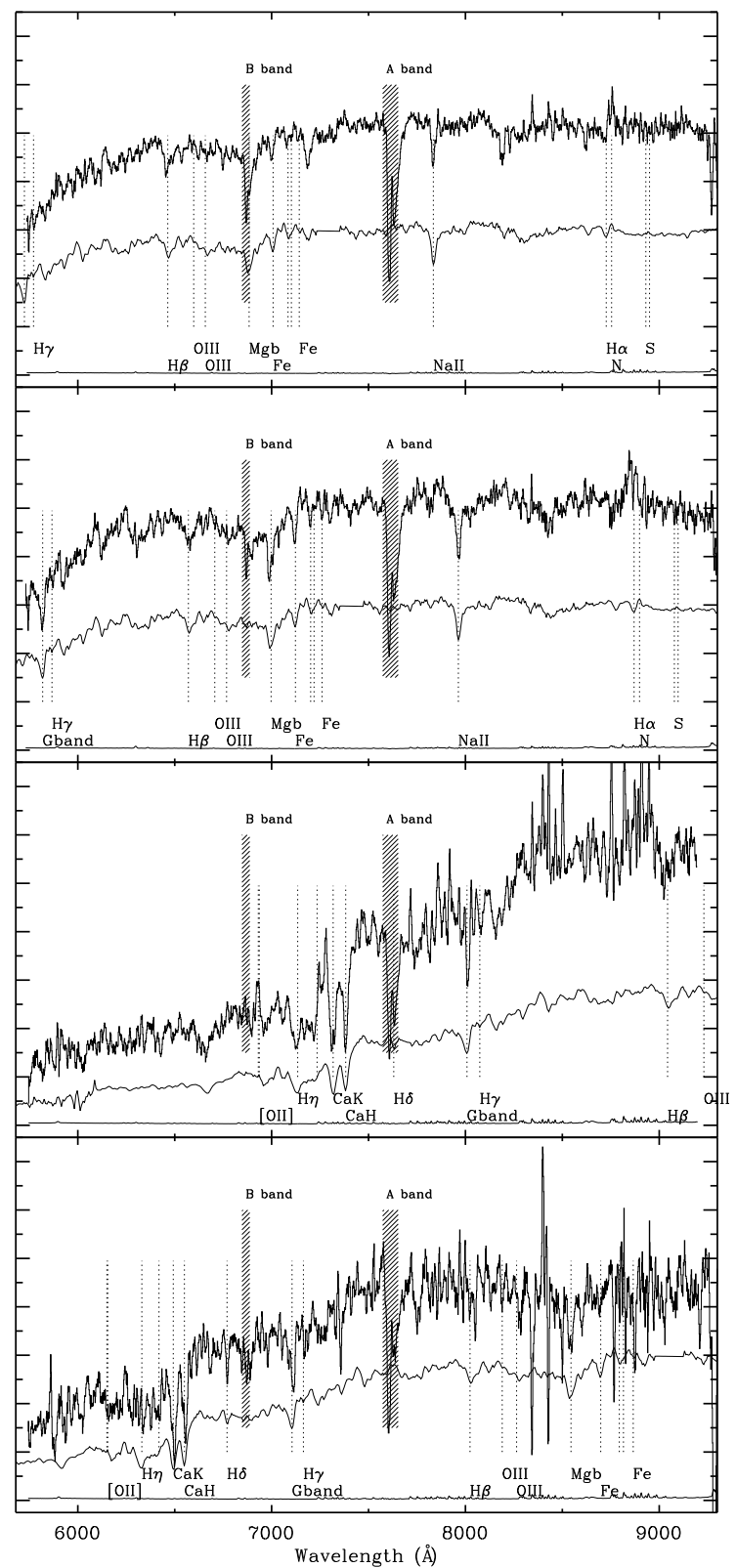

Leauthaud et al. (2007) model this distribution using the following functional form:

$$
\begin{aligned}
\operatorname{Pr}\left(z_{\mathrm{s}} \mid m\right) & \propto z_{\mathrm{s}}^{2} \exp \left[-\left(\frac{z}{z_{0}(m)}\right)^{1.5}\right] \\
z_{0}(m) & =\frac{(0.18 m-3.3)}{1.412} .
\end{aligned}
$$

Here $m$ is the AB magnitude of the source in the $\mathrm{F} 814 \mathrm{~W}$ filter. In most cases we have either a $\mathrm{F} 606 \mathrm{~W}$ magnitude from the HST image modeling, or a $g$-band magnitude from the CFHTLS image modeling: we assume that the sources, as faint blue galaxies, have spectral energy distributions consistent with flat in the $\mathrm{AB}$ magnitude system, and hence substitute our blue unlensed source magnitudes (output from the lens inversions) directly. This approximate transformation introduces a small additional uncertainty which we neglect relative to the in- 

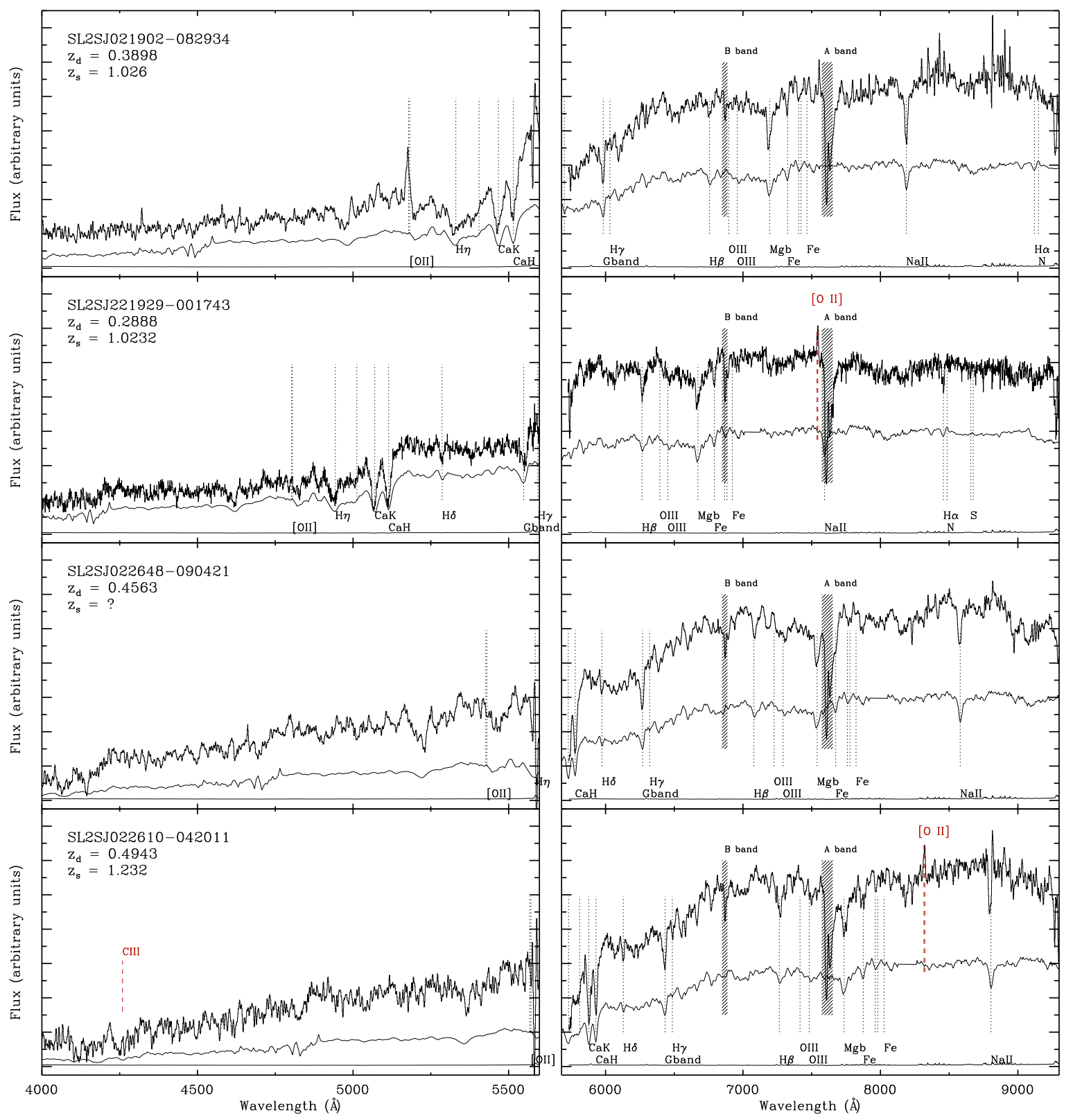

FIG. 1.- continued.

trinsic PDF width.

This model source redshift distribution was used by, among others, Gavazzi et al. (2007) and Lagattuta et al. (2010) when estimating the redshift distribution of background sources. We note that this approach is qualitatively different from that taken by Gavazzi et al. (2007) and Lagattuta et al. (2010): in their weak lensing studies they did not have a single well-defined source magnitude, but instead integrated over the number counts down to the magnitude limit. Whereas we are able to use the small amount of information that the source brightness provides. We neglect the small uncertainty in $z_{0}(m)$ and the photometric uncertainty in $m$, and truncate the density to zero at $z_{\mathrm{s}} \leq z_{\mathrm{d}}$. We then draw samples from $\operatorname{Pr}\left(z_{\mathrm{s}} \mid m\right)$ that we can then transform into distances, physical masses and so on. This gives the broader $\operatorname{PDF}, \operatorname{Pr}\left(z_{\mathrm{s}}\right)$.
While the source magnitude provides a very rough photometric redshift, we can also use the lens geometry to give a similarly rough geometric redshift. This involves multiplying the COSMOS prior $\operatorname{PDF} \operatorname{Pr}\left(z_{\mathrm{s}} \mid m\right)$ by an additional distribution describing our prior knowledge of the lens strength, as follows. In practice we do this by importance sampling the COSMOS prior (see e.g. Lewis \& Bridle 2002; Suyu et al. 2010, for descriptions of how this process works). Given that the velocity dispersion of the dark matter is approximately equal to the central stellar velocity dispersion, and the apparently universal (approximate) isothermal profile of lens galaxies (see e.g. Kochanek 1994; Koopmans et al. 2006), we can down weight some predicted $z_{\mathrm{s} \text {,pdf }}$ values based on the velocity dispersions they predict. The total deflector mass is modeled as a Singular Isothermal Ellipsoid (SIE), with a 


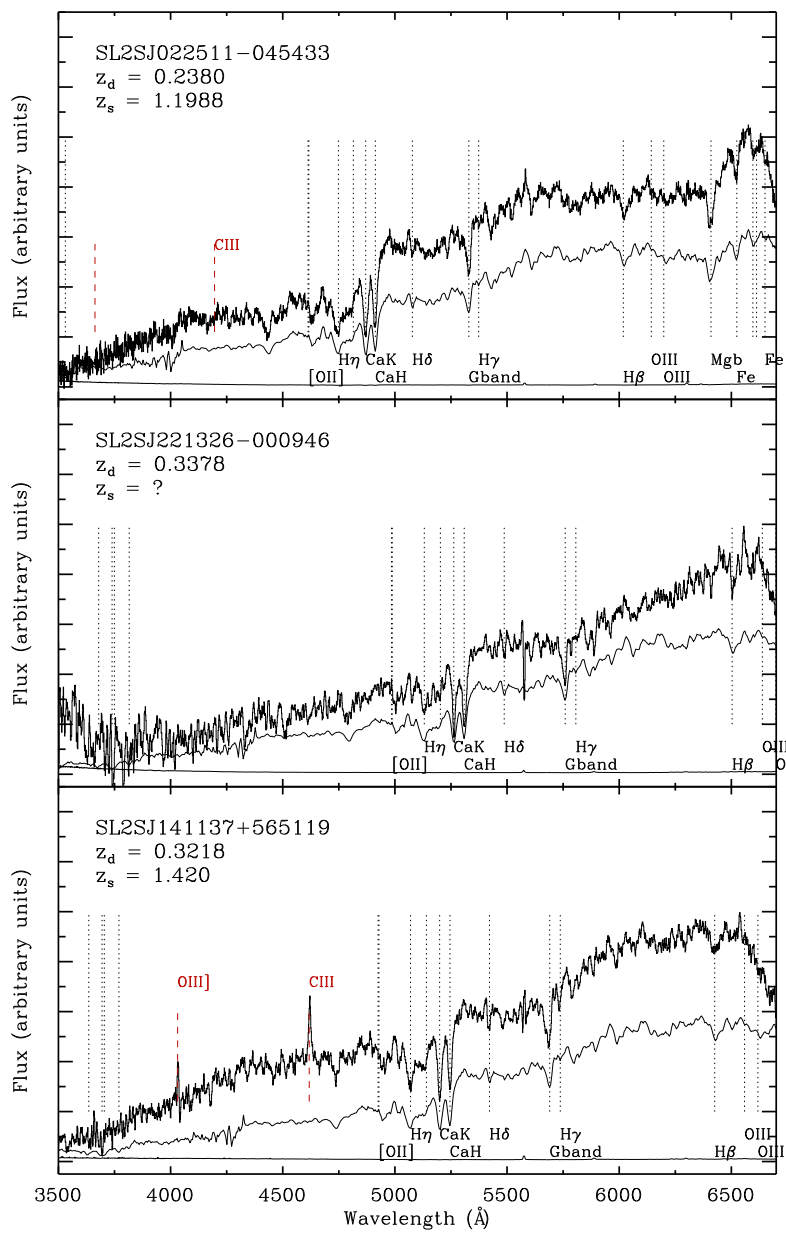

FIG. 1.- continued.

velocity dispersion

$$
\sigma_{\mathrm{SIE}}=186.2 \mathrm{~km} \mathrm{~s}^{-1} \times \sqrt{\frac{R_{\operatorname{Ein}}}{\operatorname{arcsec}} \frac{D_{\mathrm{ds}}}{D_{\mathrm{s}}}}
$$

where $D_{\mathrm{ds}}$ and $D_{\mathrm{s}}$ are the angular diameter distances between the deflector and source and observer and source, respectively. The calculated $\sigma_{\mathrm{SIE}}$ for each $z_{\mathrm{s} \text {,pdf }}$ was used to give a $\operatorname{Pr}\left(\sigma_{\text {SIE }}\right)$. A joint prior, $\operatorname{Pr}\left(z_{\mathrm{s}} \mid m\right) \operatorname{Pr}\left(\sigma_{\mathrm{SIE}}\right)$, using the additional information from $\sigma_{\text {SIE }}$ was then calculated and used to tighten the constraint on $z_{\mathrm{s}}$.

To calculate $\operatorname{Pr}\left(\sigma_{\mathrm{SIE}}\right)$, we assumed that the SL2S lens galaxies are at the high mass end of the velocity function of bright galaxies - high mass, because we know they are acting as gravitational lenses. To describe the velocity function of the SL2S lenses, the velocity dispersion fitting function determined by Sheth et al. (2003) was used. The parameters in the fitting function were determined using measurements of a large sample of earlytype galaxies drawn from the SDSS database. The fitting function was multiplied by $\sigma^{4}$ to mimic the lensing selection function (see e.g. Auger et al. 2010). However, the lenses, particularly those with measured $\sigma$, are also luminosity selected because SDSS is a flux-selected sample and more luminous objects are drawn from a large volume (Hyde \& Bernardi 2009; Auger et al. 2010). To account for the luminosity selection, the Sheth et al. fitting function was heuristically multiplied by $\sigma^{4}$. Figure 4 shows the distribution of the measured SL2S ve-

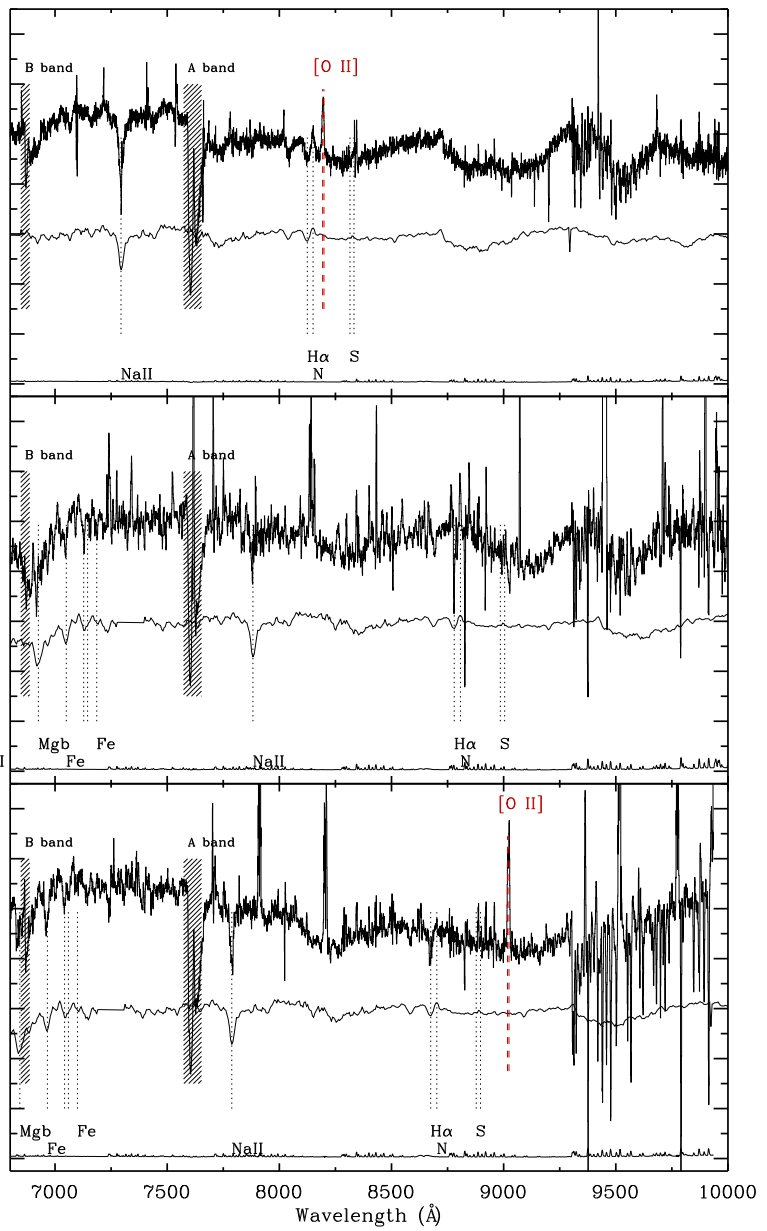

locity dispersions with the Sheth et al. fitting function, multiplied by two different selection functions, overlayed. The dashed curve shows the fitting function multiplied by $\sigma^{4}$ (mimicking the lensing selection function), while the solid curve shows the fitting function multiplied by both the lensing and luminosity selection functions. Despite the small number of objects, the mean $\sigma$ of the SL2S deflectors $\left(250 \pm 39 \mathrm{~km} \mathrm{~s}^{-1}\right)$ is in very good agreement with the peak of the solid curve $\left(243 \pm 48 \mathrm{~km} \mathrm{~s}^{-1}\right)$. The prior adopted is then the Sheth et al. fitting function multiplied by $\sigma^{8}$; the resulting distribution was then normalized and used to compute importances to weight the $z_{\mathrm{s} \text {,pdf }}$ values drawn from the COSMOS prior. We note that final result is relatively insensitive to the precise choice of the exponent, since the dominant effect is the exponential cutoff, which eliminates unrealistically high stellar velocity dispersions.

Our final prior PDF for the velocity dispersions of the SL2S galaxies is then given by:

$$
\operatorname{Pr}\left(\sigma_{\mathrm{SIE}}\right) \propto \sigma^{\alpha+7} e^{-\left(\frac{\sigma}{\sigma_{*}}\right)^{\beta}},
$$

where the best fit parameters determined by Sheth et al. (2003) for early-type galaxies are: $\sigma_{*}=88.8 \pm 17.7 \mathrm{~km} \mathrm{~s}^{-1}$, $\alpha=6.5 \pm 1.0$ and $\beta=1.93 \pm 0.22$. To account for the scatter in $f_{\mathrm{SIE}}=\sigma_{\mathrm{SIE}} / \sigma_{*}$, the function was conservatively smeared by $20 \%$. This distribution is quite broad: the assumption of an approximately isothermal mass profile does not significantly bias our results when inferring the 

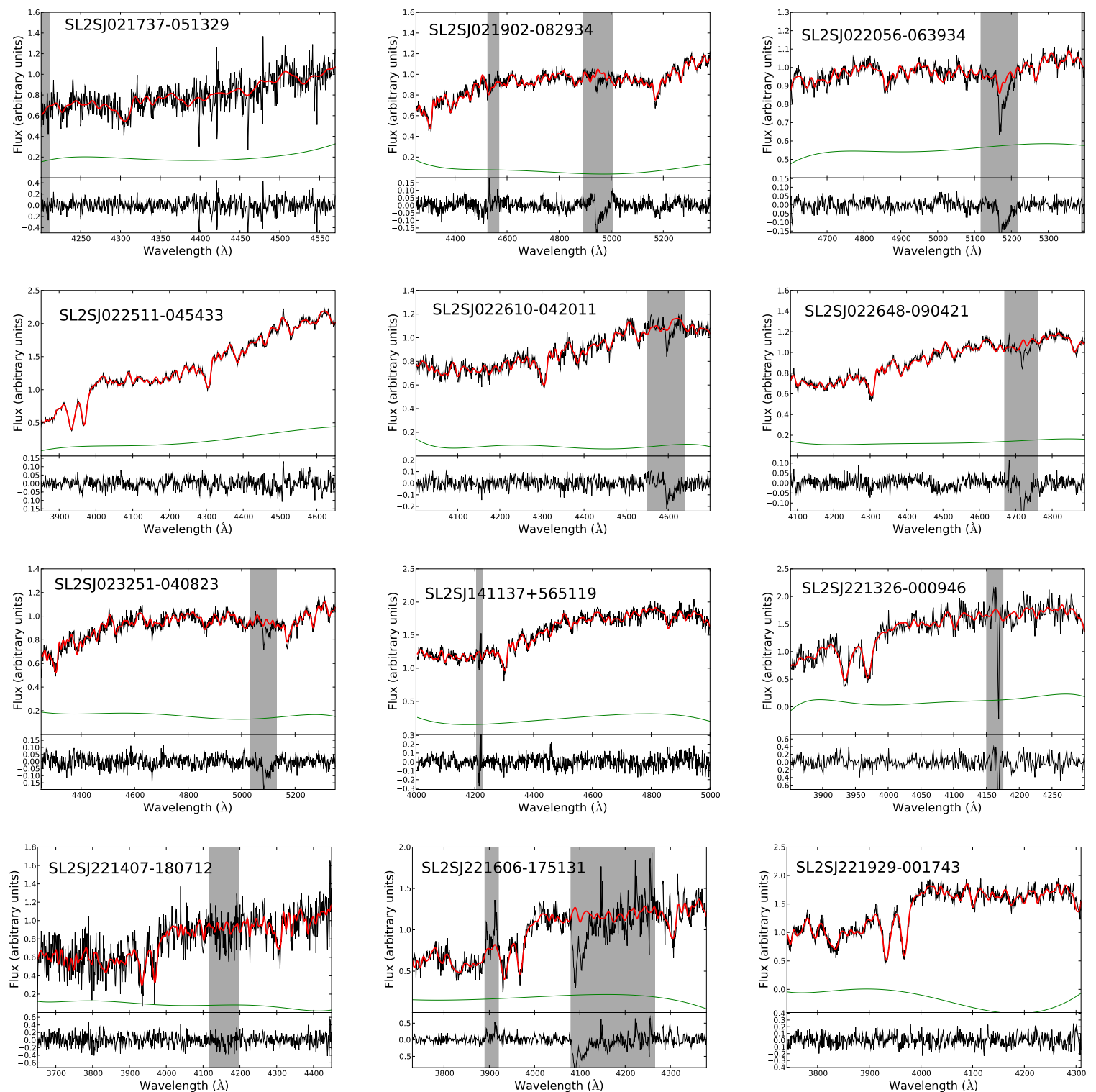

FIG. 2.- Measuring the stellar velocity dispersions of the lens galaxies, by accurate absorption line fitting. For each object, we show the relevant portion of the LRIS spectrum (black line), compared to a model generated from all 9 INDO-US templates and a sixth order polynomial continuum (in red, with the continuum alone shown in green). The grey shaded wavelength ranges were not included in the fits. The lower sub-panels show the fit residuals in each case.
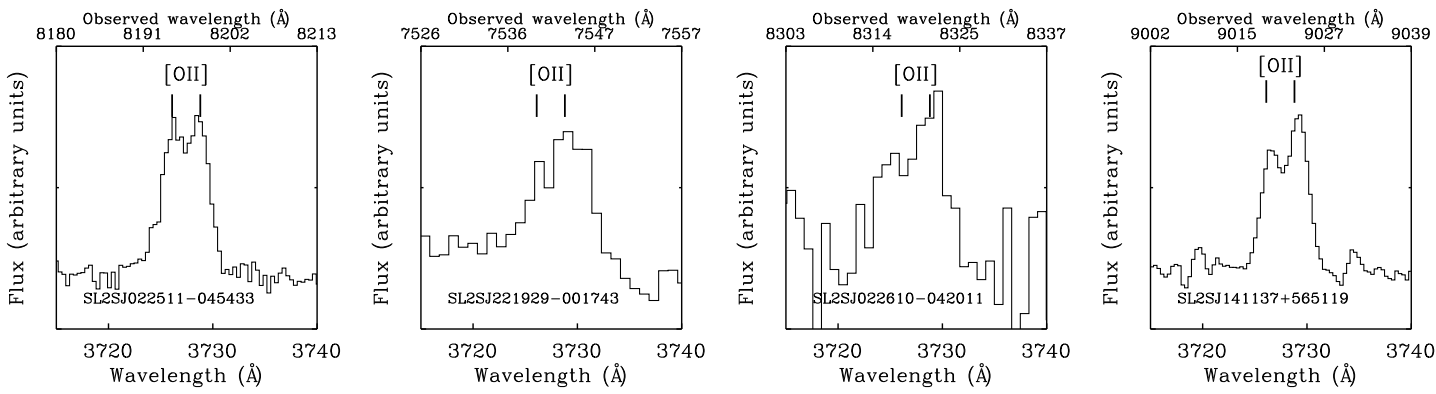

FIG. 3.- The [O II] doublet is shown for each object with a measured source redshift and observed [O II] doublet. 


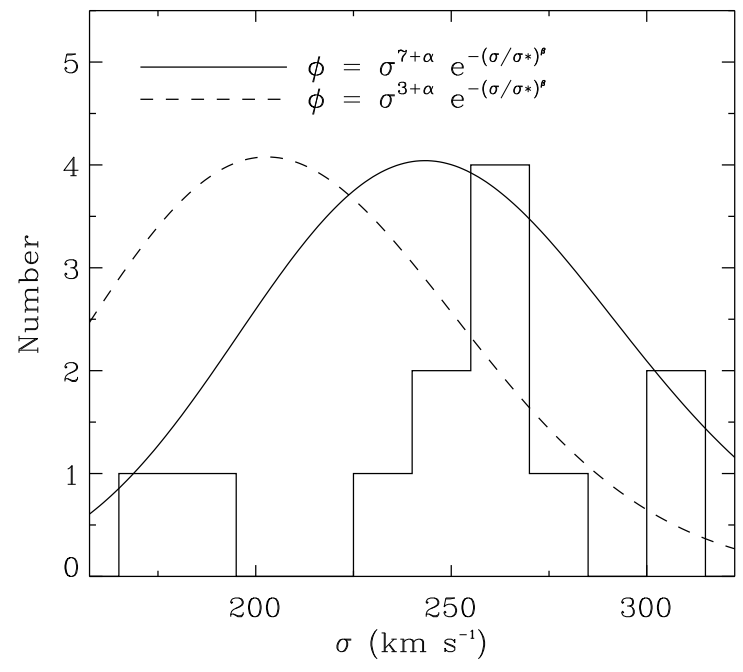

FIG. 4.- The distribution of measured velocity dispersions is shown with the Sheth et al. fitting function overlayed. The solid and dashed curves show the fitting function multiplied by $\sigma^{8}$ (lensing and luminosity selection function) and $\sigma^{4}$ (only lensing), respectively. The fitting functions were normalized to the unit area of the histogram. Note that the mean of the SL2S $\sigma$ distribution is in good agreement with the peak of the solid curve.

profile slopes of individual lenses, since we include plausible scatter on $\sigma$. Distributions of $z_{\mathrm{s}, \mathrm{pdf}}$ for the final sample of lenses are shown in Figure 5. The dashed line shows $z_{\text {s,pdf }}$ distributions from the COSMOS prior without weightings and the solid line indicates the final $z_{\mathrm{s}, \mathrm{pdf}}$ for each of the lens.

The primary effect of $\operatorname{Pr}\left(\sigma_{\mathrm{SIE}}\right)$ is to disfavor lower redshift solutions to the lens equation, that are offered by the COSMOS redshift distribution with its relatively low $z_{\mathrm{s}}$ peak, which would indicate unrealistically large stellar velocity dispersions, well above $400 \mathrm{~km} \mathrm{~s}^{-1}$. For each non-spectroscopic case, we give the median of each lens system's source redshift PDF in Table 2, along with the $16^{\text {th }}$ and $84^{\text {th }}$ percentiles as indicators of the uncertainty. The method was verified by comparing the 5 spectroscopically measured redshifts to those inferred via this procedure, using the same 5 systems. The grey vertical lines in Figure 5 show the measured source redshifts, which are in good agreement with $z_{\mathrm{s} \text {,pdf }}$ after weighting by the Sheth et al. (2003) fitting function and the selection function.

\section{PHOTOMETRIC MEASUREMENTS}

We now turn to our photometric data, in order to measure sizes and stellar masses of our lens galaxies. The latter are required in order for us to probe the dark matter fractions in the core regions of these objects, and are obtained by stellar population analysis of the CFHTLS photometry.

\subsection{Model size and magnitude estimation}

We fit an elliptically symmetric de Vaucouleurs profile (de Vaucouleurs 1948) surface brightness distributions to each of the lens galaxies in our sample, to measure the total magnitudes and effective radii. For each lens, we used the galfit software package to fit for the position, effective (half-light) radius, ellipticity, orientation angle and total magnitude.
When available, we also used the HST data that generally provide a better estimate of the deflector effective radius. In particular, the light profile in the reddest available filter should be a better tracer of the underlying stellar mass distribution profile. Otherwise we used the average effective radius of the CFHT r, i and z bands and the internal filter-to-filter r.m.s. scatter to get a precise estimate of the effective radius and its associated measurement error. Under the assumption of a spheroidal distribution of stars, with a single stellar population and no color gradients, the scatter across the filter set in each morphological parameter gives a simple estimate of the uncertainty of each parameter, and in particular on $R_{\text {eff. }}$. In most cases, the typical error on $R_{\text {eff }}$ is of order $5 \%$. Note also that $R_{\text {eff }}$ is expressed along the geometric mean axis, that is the radius of the circle enclosing the same area as the elliptical isophote enclosing half the light.

To build up a picture of each lens' spectral energy distribution (SED), with the deflector photometry devoid of light coming from the lensed background source, we follow a two-step iterative process to mask out the lensed images: first we fit models to the images with no masking, and then use the residual image to manually mask out the lensed features, before refitting the model. Apparent magnitudes are then corrected for Galactic dust extinction (Schlegel et al. 1998). Errors are dominated by systematic photometric zero point uncertainties, dust correction errors and variations of galfit results depending on the masking strategy. All together these amount to systematic errors of $\sim 0.05$ magnitudes in each CFHT bands. The resulting effective radii and magnitudes are given in Table 2.

\subsection{SED fitting methodology}

Estimates of the stellar masses were calculated using the CFHT galfit model magnitudes and composite stellar population models using a code developed by Auger et al. (2009). The code employs a Bayesian exploration of the stellar populations of galaxies using composite stellar population models produced by Bruzual \& Charlot (2003). The code takes a set of photometric data for each object $(\mathrm{g}, \mathrm{r}, \mathrm{i}, \mathrm{z})$ magnitudes and their uncertainties from the CFHT filters and the measured redshift. The free parameters of the star formation history are: the time when star formation began, the time scale of the exponential decay of the star formation rate, internal reddening due to dust extinction, and metallicity. The parameter space was then explored using a Markov Chain Monte Carlo (MCMC) routine, allowing a full determination of the posterior probability distribution function for each parameter; see Auger et al. (2009) for a comprehensive description of the method. We employ uniform priors for all of the model parameters (including the metallicity) due to the absence of any well-calibrated priors for the stellar population model parameters of massive galaxies at the redshifts of these lenses.

The code was run for both Salpeter (1955) and Chabrier (2003) initial mass functions (IMFs). The masses derived from the Salpeter IMF were a factor of $\sim 1.7$ greater than the masses derived using the Chabrier IMF. In a recent study of 56 gravitational lenses identified by the SLACS survey, Treu et al. (2010) found that for massive early-type galaxies, the Salpeter IMF provides stellar masses in approximate agreement with 

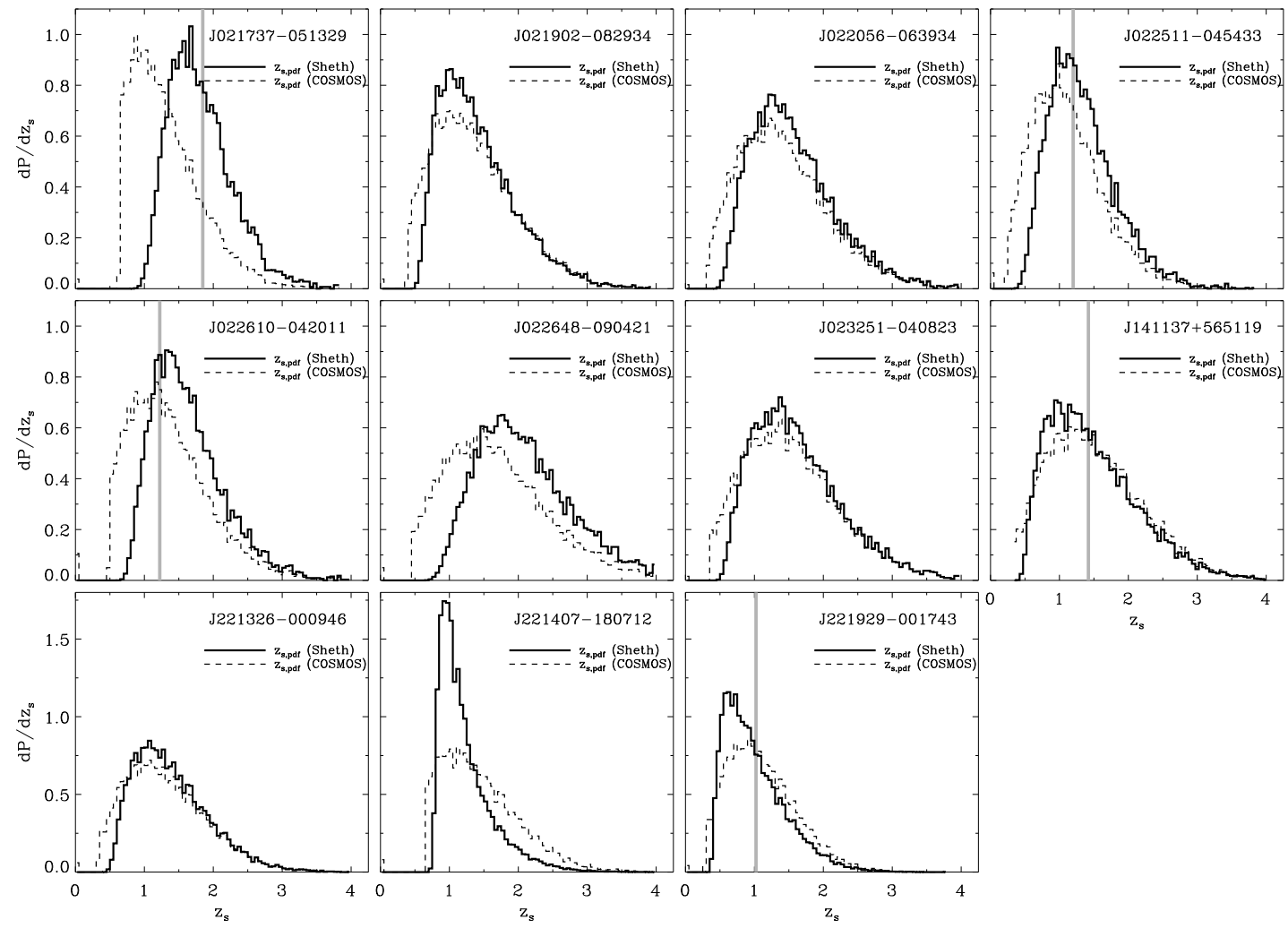

FIG. 5.- The $z_{\mathrm{s}, \mathrm{pdf}}$ distributions are shown for the final sample of lenses. The dashed histogram shows the source redshift inferred from the COSMOS distribution. The thick histogram shows the final $z_{\mathrm{s}, \mathrm{pdf}}$, where the inferred source redshifts were weighted by the Sheth et al. fitting function (multiplied by $\sigma^{8}$ ), as discussed in the text. For lenses with a measured $z_{\mathrm{s}}$, the grey vertical line shows the measured source redshift. Note that the PDFs have been normalized to unit area and that y-axis on the bottom row is on a different scale.

those inferred by lensing and dynamical models, whereas the Chabrier IMF gives (on average) an underestimate. However, the IMF normalization may be mass dependent being more similar to a Chabrier IMF for the lower mass systems in the SLACS sample, with $\sigma \sim 200 \mathrm{~km} \mathrm{~s}^{-1}$ (Barnabe et al. 2010; Auger et al. 2010; see also Cappellari et al. 2006, 2009). For simplicity, we adopt the Salpeter IMF normalization, although they can easily be converted to other normalizations, by multiplying by an appropriate factor (e.g. Auger et al. 2009). Total stellar masses for a Salpeter IMF are given in Table 3.

LSD stellar masses were also calculated using the method described above with photometry from Koopmans \& Treu $(2002,2003)$ and Treu \& Koopmans (2004). This method could only be applied to 4 of the 5 LSD lenses, as photometry in multiple bands is required. Using this method we inferred total stellar masses (in units of $\left.10^{11} M_{\odot}\right)$ of: $5.01 \pm 2.06,2.24 \pm 0.58,4.26 \pm 0.95$ and $2.49 \pm 0.82$ for $\mathrm{H} 1417+526, \mathrm{H} 1543+535, \mathrm{MG} 2016+112$ and 0047-281, respectively.

\section{LENS MODELING}

The modeling of our high resolution HST images is described in detail in Paper I. Here, we briefly outline the procedure followed and describe the relevant output parameters that we use in our joint analysis. Following standard image reduction, the bright lens galaxies were subtracted from postage stamp images of the lenses, iteratively masking the lensed arcs. The remaining residual image was fitted using a flexibly parametrized source (an elliptical exponential profile with free position, ellipticity, orientation, size and flux) traced through a simple ellipti- cally symmetric lens potential. We model each lens mass distribution using a Singular Isothermal Ellipsoid (SIE) model (Kormann et al. 1994), with centroid fixed at the centroid of the galfit model lens light distribution. The lens has three free parameters: Einstein radius $R_{\text {Ein }}$, orientation $\phi$ and axis ratio $q$. In all cases the lens redshift is known spectroscopically, allowing us to work with Einstein radius in $\mathrm{kpc}$.

The parameters of each lens were inferred by exploring their posterior PDF using a Markov Chain Monte Carlo (MCMC) sampler. While the samples do characterize the posterior PDF as required, the simplicity of the lens model and the idealized source light distribution mean that, in practice, the posterior width does not provide a good estimate of the uncertainty on, for example, the Einstein radius. Instead, we use independent reconstructions in several bands (which we have for a subset of the lenses) to estimate the uncertainties (see e.g. Marshall et al. 2007), which are found to be about $5 \%$ on $R_{\text {Ein }}$.

The properties of the lens models are discussed in detail in Paper I; here we are concerned only with the combination of the lensing mass with the dynamical mass from our spectroscopic measurements. With this in mind, the definition of elliptical radius in the lens modeling was chosen to preserve the enclosed mass with circular apertures, adopting the same definition as the SLACS survey (Koopmans et al. 2006; Bolton et al. 2008b; Auger et al. 2009). The mass enclosed within the Einstein radius, $M_{\text {Ein }}$, is essentially independent of the profile of the lens density profile: although we used an SIE model to infer $M_{\text {Ein }}$, we can treat it as a profile-independent, circularly symmetric enclosed mass and then combine it 


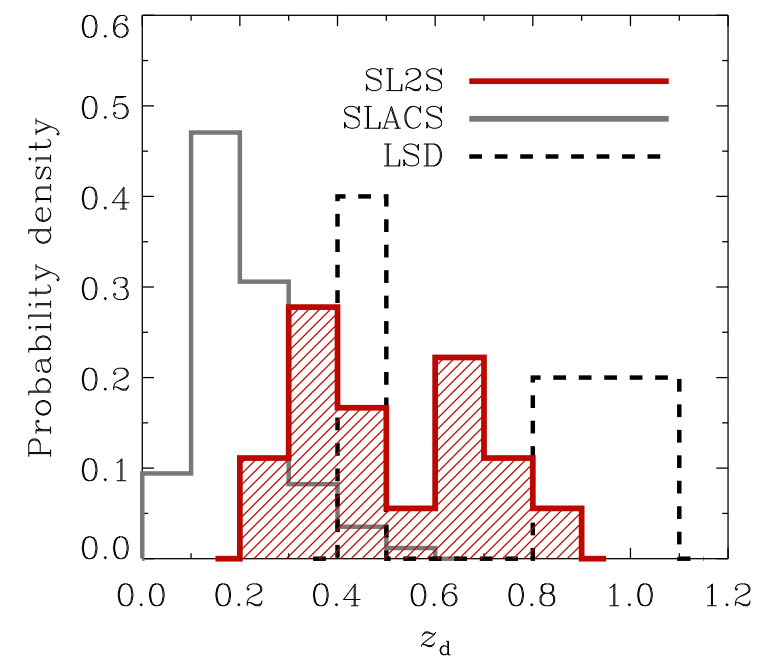

FIG. 6.- Redshift distributions of the SL2S, SLACS and LSD surveys. All three distributions have been normalized to one.

with a dynamical spherical mass estimate in order to infer the total density profile slope (see Section 7.4 below). For more discussion of the accuracy of this assumption see Auger et al. (2009).

Three systems J140614+520252, J220629+005728 and J221606-175131, were observed with LRIS before attempting to perform a lens model. But subsequently we were not able to find a satisfactory lens model and we failed in measuring their Einstein radius essentially because HST imaging is not available for them yet. Deflector photometry along with effective radius and redshift are nevertheless reported in Table 2. A final conclusion on their lensing nature is left for future work (see also Paper I for further discussion).

\section{CHARACTERIZATION OF THE SL2S SAMPLE}

In this section we explore the basic properties of the SL2S lens galaxies, and compare them with two previously studied samples of lenses. The SLACS sample is a lower redshift sample, $0.08<z_{\mathrm{d}}<0.51$, of 85 lenses from the Sloan Digital Sky Survey (Bolton et al. 2006, 2008a). The LSD survey measured the internal kinematics of 5 early-type lens galaxies over a large range of redshifts, $0.48<z_{\mathrm{d}}<1.00$, and masses (Treu \& Koopmans 2004).

\subsection{The redshift distributions of SL2S lenses}

It is instructive to compare the distribution of lens redshifts in the SL2S sample, described in the preceding section, with those of the lower and higher redshift reference samples, SLACS and LSD, respectively. In Figure 6, we show histograms of deflector redshifts for the SL2S (red), SLACS (grey) and LSD (black) samples. We see that, as anticipated, the SL2S lenses lie at higher redshift than the SLACS sample. The median and $68 \%$ confidence intervals are $z_{\mathrm{d}}=0.494_{-0.16}^{+0.16}, z_{\mathrm{s}}=1.199_{-0.18}^{+0.22}$ for the SL2S sample, $z_{\mathrm{d}}=0.189_{-0.066}^{+0.096} z_{\mathrm{s}}=0.606_{-0.157}^{+0.201}$ for SLACS and $z_{\mathrm{d}}=0.810_{-0.066}^{+0.096} z_{\mathrm{s}}=3.263_{-0.61}^{+0.61}$ for LSD.

\subsection{Comparison to previous lens samples}

The measured stellar velocity dispersions and effective radii provide us with a means to compare the SL2S, SLACS and LSD samples, to assess whether we are

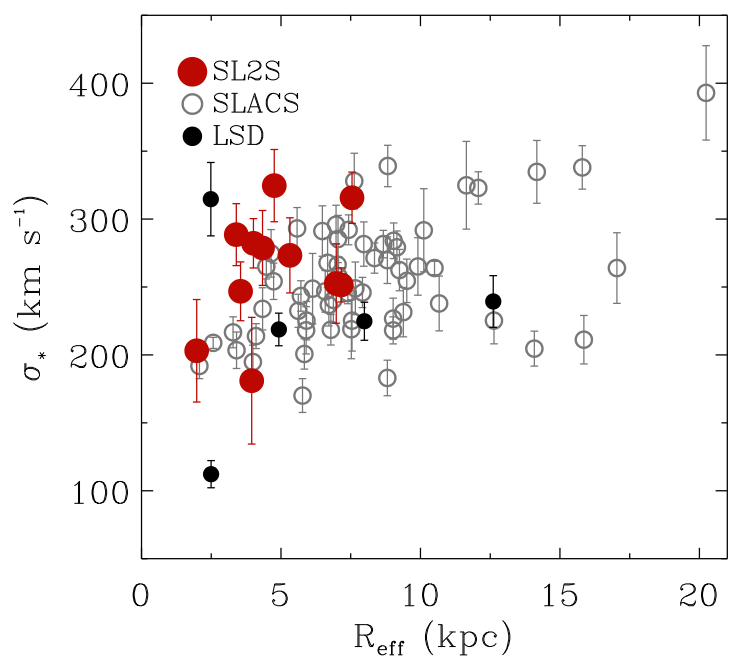

FIG. 7.- Stellar velocity dispersions are plotted against the effective radius in the $\mathrm{V}$ band. The SL2S, SLACS (Auger et al. 2009) and LSD (Treu \& Koopmans 2004) data points are shown in red, grey and black, respectively. Note that $R_{\text {eff }}$ is measured at the intermediate axis and the stellar velocity dispersions have been corrected to $\sigma_{e 2}$.

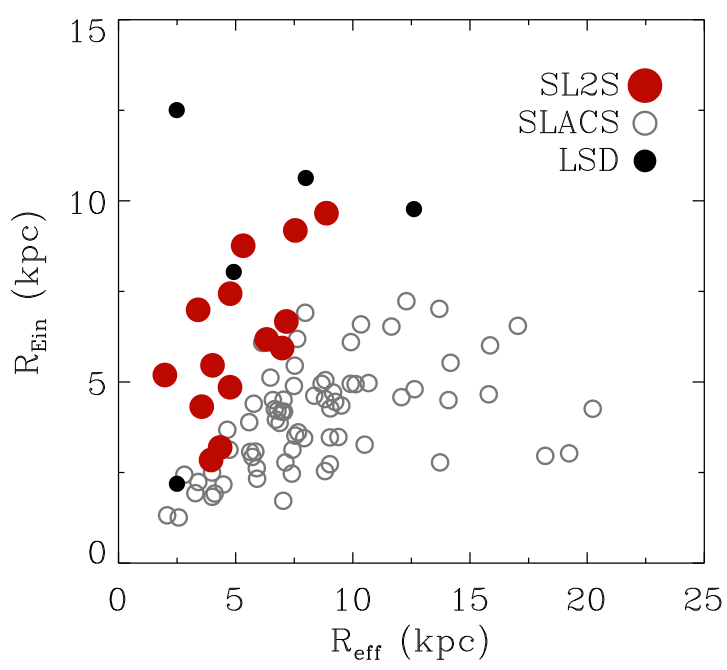

FIG. 8. - The effective radius is plotted against the Einstein radius. The SL2S, SLACS and LSD points are shown in red, grey and black, respectively. Typical uncertainties on $R_{\text {Ein }}$ and $R_{\text {eff }}$ are $5 \%$ and $10 \%$, respectively.

studying the same types of galaxies. In Figure 7, we plot our spectroscopic velocity dispersions, $\sigma$ against the ef-

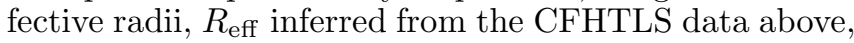
in order to compare the types of galaxies selected in the SL2S, SLACS and LSD samples. The stellar velocity dispersions are consistent between the samples, while the effective radii of the SLACS galaxies are slightly higher. Note that the stellar velocity dispersions have been normalized to a standard aperture, $\sigma_{\mathrm{e} 2}$ for all samples.

To compare the samples quantitatively, we applied the 2D Kolmogorov-Smirnov test in the $\sigma-R_{\text {eff }}$ plane. We found the KS statistic to be 0.28 , and the significance level to be $91 \%$. Given this high significance, we conclude that we are drawing from the same population of galaxies, despite the marginal tendency towards larger sizes in the SLACS sample. 
Figure 8 shows the distribution of $R_{\text {eff }}$ and $R_{\text {Ein }}$ for the SL2S, SLACS and LSD samples. The higher redshift samples (SL2S and LSD), have much higher physical $R_{\text {Ein }}$ values while the effective radii are slightly smaller, as discussed above. The difference in $R_{\text {Ein }}$ is partly due to the method used to select the lens candidates: resolvable SL2S ring radii of $0.5-2$ arcsec correspond to larger physical radii than those of the SLACS sample. SLACS lenses were pre-selected to be bright SDSS target galaxies. This selection method ensures that they lie at relatively low redshift, which in turn means that the characterizing angular size of Einstein rings translates to a smaller physical Einstein radii for the SLACS lenses than the SL2S lenses. Furthermore, the source redshifts are on average significantly higher for the LSD and SL2S samples, implying larger Einstein Radii for the same mass distribution.

We conclude that we do seem to be measuring similar types of galaxies, with similar sizes, in a similar mass range: the SLACS and SL2S lenses are consistent with having been drawn from the same population. However, the different lens geometry (due to the difference in redshift between the samples) means that we are sampling the mass density profile at a larger radius, which is approximately a factor of 2 times greater compared to the SLACS lenses.

\section{THE INTERNAL MASS STRUCTURE OF SL2S LENSING GALAXIES}

In this section we use our new spectroscopic measurements to carry out a joint lensing and dynamics analysis of the SL2S sample, estimating the total mass density profile slope and dark matter fraction of each individual lens galaxy. We first describe the simple model within which we work, and then present our inferences of these two key parameters.

\subsection{Power-law density profiles}

We choose to work in the context of a sphericallysymmetric power law total mass density profile model (e.g. Treu \& Koopmans 2002; Koopmans et al. 2006; Suyu et al. 2010):

$$
\begin{aligned}
\rho_{t o t}(r) & \propto r^{-\gamma^{\prime}} \\
\Sigma(R) & \propto R^{1-\gamma^{\prime}} .
\end{aligned}
$$

As in the lens modeling, we use capitalized $R$ to denote projected radius. We can normalize these power law profiles in terms of $M_{\text {Ein }}$, the robustly-estimated, profileindependent Einstein Mass, from Section 5 above: our approach is to use the well-measured Einstein radii and Einstein masses from the lens models of Paper I, and reinterpret them within the context of this spherical powerlaw model.

The spherical power law model allows us to predict the observed spectroscopic velocity dispersion of the tracer stellar population, via the Jeans equation (see, e.g. Treu \& Koopmans 2004; Koopmans et al. 2006; Auger et al. 2010; Suyu et al. 2010). Normalizing the surface density and integrating to the (observable) Einstein radius $R_{\text {Ein }}$, we find that

$$
\Sigma(R)=\frac{\left(3-\gamma^{\prime}\right)}{2} \Sigma_{\text {crit }}\left(\frac{R}{R_{\text {Ein }}}\right)^{1-\gamma^{\prime}}
$$

$$
\rho_{\text {tot }}(r)=\frac{\left(3-\gamma^{\prime}\right)}{2 \pi^{1 / 2}} \frac{\Gamma\left(\frac{\gamma^{\prime}}{2}\right)}{\Gamma\left(\frac{\gamma^{\prime}-1}{2}\right)} \frac{\Sigma_{\text {crit }}}{R_{\text {Ein }}}\left(\frac{r}{R_{\text {Ein }}}\right)^{-\gamma^{\prime}} .
$$

Since $M_{\text {Ein }}=\pi R_{\operatorname{Ein}}^{2} \Sigma_{\text {crit }}$, we can choose the two parameters of our model to be $M_{\text {Ein }}$ and $\gamma^{\prime}$. In the next subsection we outline how the stellar velocity dispersion is predicted from these parameters.

\subsection{Stellar dynamics analysis}

As described by Suyu et al. (2010) and Auger et al. (2010), we predict the stellar velocity dispersion at the appropriate aperture radius using the spherical Jeans equation. Qualitatively we use the power law profile to compute the total spherical mass enclosed within radius $r$, and assume the tracer stars follow a Hernquist distribution with scale radius related to the measured effective radius, and then integrate to predict the stellar velocity dispersion, $\sigma\left(M_{\operatorname{Ein}}, \gamma^{\prime}\right)$. We direct the reader to Section 2.3 of Suyu et al. (2010) for all relevant equations. We assume isotropic orbits for all of the systems, which is approximately found to be the case from a more detailed analysis of the resolved kinematics of several SLACS lenses (Barnabè et al. 2009).

\subsection{Joint analysis}

We propagate the uncertainties in the Einstein mass (which can be significant, for systems with no spectroscopic source redshift), and the spectroscopic velocity dispersion, in the following way. The output of the lens modeling procedure for each lens is the posterior probability distribution for the Einstein mass given the $H S T$ image data $\mathbf{d}, \operatorname{Pr}\left(M_{\text {Ein }} \mid \mathbf{d}, z_{\mathrm{d}}, z_{\mathrm{s}}\right)$, characterized as a set of sample $M_{\text {Ein }}$ values - this can be viewed as the prior $\mathrm{PD}$ for $M_{\text {Ein }}$ for the joint analysis. The analysis of that lens' spectrum yields the likelihood, $\operatorname{Pr}\left(\sigma^{\text {obs }} \mid M_{\text {Ein }}, \gamma^{\prime}\right)$, which we assume to be Gaussian in $\sigma^{\text {obs }}$, with mean equal to the Jeans prediction $\sigma\left(M_{\text {Ein }}, \gamma^{\prime}\right)$ from the previous subsection.

The posterior PDF that we seek for each lens is then

$$
\begin{aligned}
\operatorname{Pr}\left(M_{\text {Ein }}, \gamma^{\prime} \mid \sigma^{\text {obs }}, \mathbf{d}, z_{\mathrm{d}}, z_{\mathrm{s}}\right) \propto & \operatorname{Pr}\left(\sigma^{\text {obs }} \mid M_{\text {Ein }}, \gamma^{\prime}\right) \\
& \cdot \operatorname{Pr}\left(M_{\text {Ein }} \mid \mathbf{d}, z_{\mathrm{d}}, z_{\mathrm{s}}\right) \\
& \cdot \operatorname{Pr}\left(\gamma^{\prime}\right) .
\end{aligned}
$$

Since we are only working in the context of a single model, we do not compute the evidence to normalize the right-hand side of this equation; all we need are samples drawn from the posterior $\mathrm{PDF} \operatorname{Pr}\left(M_{\text {Ein }}, \gamma^{\prime} \mid \sigma^{\text {obs }}\right)$.

For the prior on the slope $\gamma^{\prime}$ we assume a uniform distribution with limits of -1.2 and -2.8 , which we find to be broad enough to enclose all the likelihood (and are in fact close to the mathematical limits required for the normalizability of the profile). We draw an equal number of sample $\gamma^{\prime}$ values from this uniform distribution to match the $M_{\text {Ein }}$ samples, and then use the likelihood evaluated at each 2-dimensional sample position as weights: for each sample $\left\{M_{\operatorname{Ein}}, \gamma^{\prime}\right\}$ we solve the Jeans equation to calculate $\sigma\left(M_{\text {Ein }}, \gamma^{\prime}\right)$, and then evaluate the Gaussian function $\operatorname{Pr}\left(\sigma^{\mathrm{obs}} \mid M_{\text {Ein }}, \gamma^{\prime}\right)$. (For more details on importance sampling, see the appendix of Suyu et al. 2010.) This set of weighted samples can then be used to compute integrals over the posterior, confidence limits, histograms to represent the marginalized distributions and so on. We present these inferences in the following two sections, and summarize our numerical results in Table 3. 


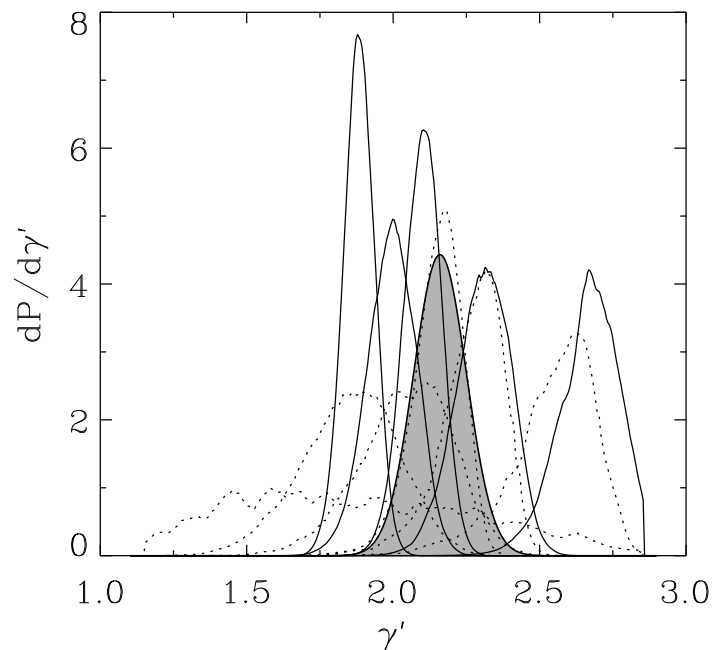

FIG. 9.- Posterior probability distributions for $\gamma^{\prime}$ using a uniform prior. The solid and dashed curves show $\gamma^{\prime}$ distributions for individual lenses with and without measured source redshifts, respectively. The shaded region indicates the posterior PDF for the mean of the Gaussian distribution from which the sample was inferred to have been drawn: $\left\langle\gamma^{\prime}\right\rangle=2.16_{-0.09}^{+0.09}$. Note that the distributions have been normalized to unit area.

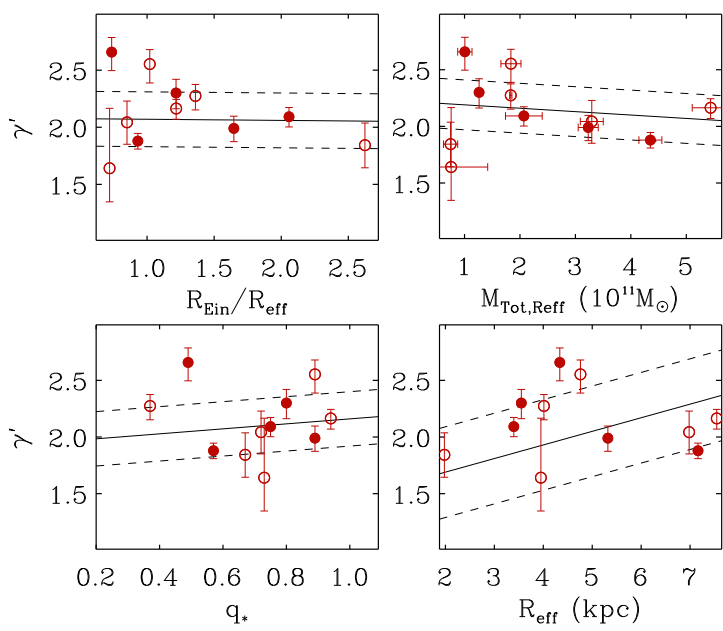

FIG. 10. - The slope of the density profile is plotted against Einstein radius, projected mass within the Einstein radius, stellar ellipticity and effective radius. The error bars show the $16^{\text {th }}$ and $84^{\text {th }}$ percentiles. The solid line shows the linear best fit to the data and the dashed lines indicate the scatter.

\subsection{The density profile slope $\gamma^{\prime}$ from lensing and stellar dynamics}

In total we have 11 SL2S lenses with both lensing and dynamical mass estimates. In Figure 9 we show the posterior probability distributions for the logarithmic density profile slope $\gamma^{\prime}$, resulting from the joint lensing and dynamics analysis. We see that the mean of the population lies at $\left\langle\gamma^{\prime}\right\rangle=2.16_{-0.09}^{+0.09}$, and is shown by a shaded region in Figure 9. The intrinsic (Gaussian) scatter of the sample, inferred assuming Gaussian errors on the individual $\gamma^{\prime}$ values, is $S_{\gamma^{\prime}}=0.25_{-0.07}^{+0.10}$. For each of the 11 lenses in the final sample, the median $\gamma^{\prime}$ (with $16^{\text {th }}$ and $84^{\text {th }}$ percentiles) is given in Table 3

We explore how $\gamma^{\prime}$ varies with: $R_{\text {Ein }} / R_{\text {eff }}$, total mass within $R_{\text {Ein }}$, axis ratio and effective radius of the lenses in Figure 10. In each panel, the solid line shows the linear best fit to the data and the dashed lines show the scatter. The gradients of the fits, including $16^{\text {th }}$ and $84^{\text {th }}$ percentiles are:

$$
\begin{aligned}
d \gamma^{\prime} / d\left(R_{\text {Ein }} / R_{\text {eff }}\right) & =-0.01_{-0.11}^{+0.21} \\
d \gamma^{\prime} / d M_{R_{\text {eff }}} & =-0.03_{-0.05}^{+0.17} \\
d \gamma^{\prime} / d q_{*} & =0.22_{-0.17}^{+0.29} \\
d \gamma^{\prime} / d R_{\text {eff }} & =0.12_{-0.07}^{+0.14}
\end{aligned}
$$

with $M_{R_{\text {eff }}}$ in units of $10^{11} M_{\odot}$, and $R_{\text {eff }}$ in kpc. The large uncertainties on the gradients indicate negligible trends between $\gamma^{\prime}$ and these variables.

As discussed in Section 9, cosmic evolution of $\gamma^{\prime}$ could be mimicked by a dependence on $R_{\text {Ein }} / R_{\text {eff }}$. However, a negligible correlation between $\gamma^{\prime}$ and $R_{\text {Ein }} / R_{\text {eff }}$ suggests that this is unlikely.

We find no correlation between $\gamma^{\prime}$ and either $R_{\text {eff }}$ or the projected mass inside $R_{\text {eff }}$, indicating that $\gamma^{\prime}$ is independent of galaxy size and mass.

\subsection{The dark matter fraction in the SL2S lenses}

We now turn to our second key parameter: the dark matter mass fraction, $f_{\mathrm{DM}}$ in the cores of lens galaxies. Following the SLACS analyses of Koopmans et al. (2006) and Auger et al. (2009), we use $R_{\text {eff }} / 2$ as the fiducial aperture radius for dark matter fraction estimation. The stellar masses calculated in Section 4.2 are total stellar masses. These must be corrected to the mass within our fiducial aperture by integrating (under the assumption that stellar surface density follows surface brightness) the de Vaucouleurs profile, which is given by:

$$
I(r)=I_{\text {eff }} \mathrm{e}^{-7.67\left[\left(\frac{r}{R_{\text {eff }}}\right)^{1 / 4}-1\right] .}
$$

The fraction of mass enclosed within $R_{\text {eff }} / 2$ is therefore given by:

$$
\frac{M_{*}\left(R_{\mathrm{eff}} / 2\right)}{M_{*, \text { total }}}=\frac{\int_{0}^{R_{\mathrm{eff}} / 2} I(r) r d r}{\int_{0}^{\infty} I(r) r d r}=0.320 .
$$

We obtained total masses within the same radius by integrating the power law total surface density profile:

$$
\frac{M\left(R_{\mathrm{eff}} / 2\right)}{M_{\mathrm{Ein}}}=\left(\frac{R_{\mathrm{eff}} / 2}{R_{\mathrm{Ein}}}\right)^{3-\gamma^{\prime}} .
$$

Total masses within the Einstein radius and total stellar masses are given in Table 3. The dark matter fraction is then defined by:

$$
f_{\mathrm{DM}}=1-\frac{M_{*}\left(R_{\mathrm{eff}} / 2\right)}{M\left(R_{\mathrm{eff}} / 2\right)} .
$$

We do not just have a single number for each of $M_{*}\left(R_{\text {eff }} / 2\right)$ and $M\left(R_{\text {eff }} / 2\right)$; rather, we have probability distributions for each. We combine these to form the posterior PDF for $f_{\mathrm{DM}}$, by applying the formulae in Equations 12, 13 and 14 to each sample $\left\{M_{*}, M\right\}$ drawn from the product of $\operatorname{Pr}\left(M_{*}\right)$ (from the SED modeling) and $\operatorname{Pr}(M)$ (from the lensing plus dynamics joint analysis) - since these PDFs are independent, the members of each $\left\{M_{*}, M\right\}$ pair can be drawn randomly from each 


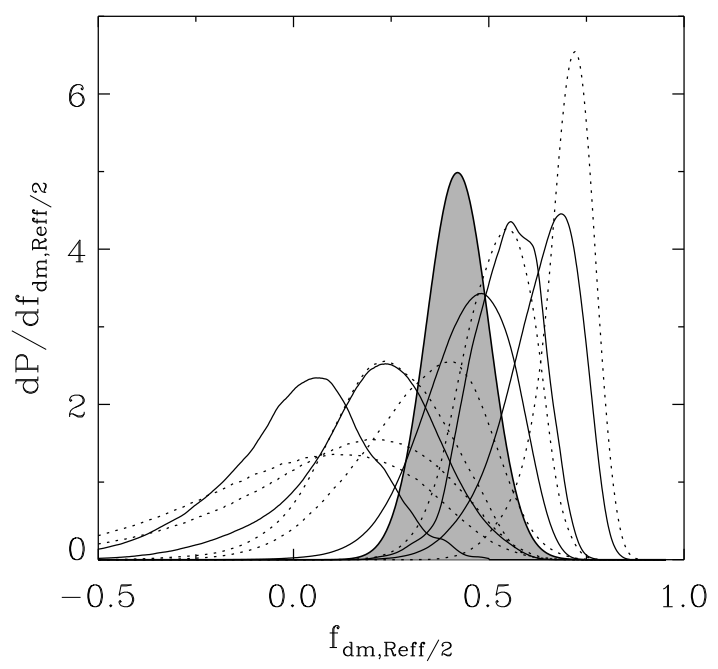

FIG. 11.- Posterior probability distributions for the dark matter fraction in the SL2S lenses. The dark matter distributions of lenses with measured source redshifts are shown in a solid line. The dashed lines show the distributions for lenses with no measured source redshift. Note that the distributions have been normalized to unit area. The shaded region indicates the posterior PDF for the mean of the Gaussian distribution from which the sample was inferred to have been drawn: $\left\langle f_{\mathrm{DM}}\right\rangle=0.42_{-0.08}^{+0.08}$.

individual ensemble. The resulting PDF for $f_{\mathrm{DM}}$ (visualized as a histogram of $f_{\mathrm{DM}}$ samples) can have significant probability at $f_{\mathrm{DM}}<0$, due to the uncertainty in each mass estimate, and the fact that our model only enforces positivity for the total and stellar mass distributions, not their difference. For consistency with the SLACS analysis, we allow negative values of $f_{\mathrm{DM}}$ (equivalent to $\left.M_{*}>M\right)$. We found that truncating the PDFs at $f_{\mathrm{DM}} \geq 0$ for the SL2S lenses did not significantly affect the numerical results.

In Figure 11 we show the resulting dark matter fraction posterior probability distribution for each SL2S lens, assuming a Salpeter IMF. The median and $16^{\text {th }}$ and $84^{\text {th }}$ percentiles of each dark matter fraction distribution are given in Table 3. We see that the mean of the population lies at $0.42_{+0.08}^{+0.08}$, as shown by the shaded region. This distribution has an intrinsic width $0.20_{-0.07}^{+0.09}$. Our results are sensitive to the choice of a universal Salpeter IMF in the stellar populations analysis. If we instead assert a universal Chabrier IMF, we find that the mean dark matter fraction is $0.68_{+0.06}^{+0.04}$, while the scatter is essentially unchanged $\left(0.11_{-0.04}^{+0.06}\right)$. As expected, a Chabrier IMF predicts a higher dark matter fraction, as shown in Table 3.

In Figure 12 we show how the SL2S lens dark matter fractions vary with: $R_{\text {Ein }} / R_{\text {eff }}$, the total mass within $R_{\text {eff }}$, axis ratio, density profile slope, and $R_{\text {eff }}$. In each panel, the solid line shows the linear best fit to the data and the dashed lines show the scatter. The gradients of the fits, including $16^{\text {th }}$ and $84^{\text {th }}$ percentiles are:

$$
\begin{aligned}
d f_{\mathrm{DM}} / d\left(R_{\text {Ein }} / R_{\text {eff }}\right) & =-0.02_{-0.12}^{+0.14} \\
d f_{\mathrm{DM}} / d M_{R_{\text {eff }}} & =0.034_{-0.045}^{+0.048} \\
d f_{\mathrm{DM}} / d q_{*} & =0.23_{-0.32}^{+0.29} \\
d f_{\mathrm{DM}} / d R_{\mathrm{eff}} & =0.08_{-0.08}^{+0.10} \\
d f_{\mathrm{DM}} / d \gamma^{\prime} & =0.40_{-0.12}^{+0.31},
\end{aligned}
$$
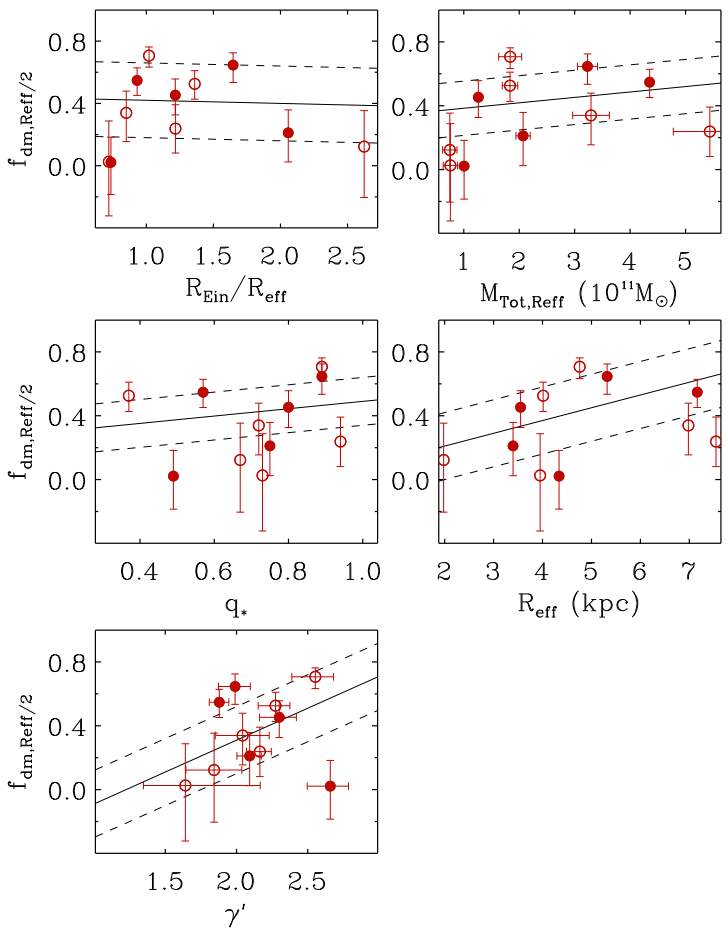

FIG. 12.- Dark matter fraction plotted against $R_{\text {Ein }} / R_{\text {eff }}$, the total mass within $R_{\text {eff }}$, axis ratio, total mass density profile slope, and $R_{\text {eff }}$. The error bars show the $16^{\text {th }}$ and $84^{\text {th }}$ percentiles. The solid line shows the linear best fit to the data and the dashed lines indicate the scatter.

with $M_{R_{\text {eff }}}$ in units of $10^{11} M_{\odot}, R_{\text {eff }}$ in kpc and $f_{\mathrm{DM}}$ is the dark matter within $R_{\text {eff }} / 2$. There is evidence for a correlation between $f_{\mathrm{DM}}$ and $\gamma^{\prime}$. For all other variables, the large uncertainties on the gradients indicate that there is only a negligible trend with $f_{\mathrm{DM}}$.

\section{COSMIC EVOLUTION}

We now investigate evolution in the properties of massive galaxies using our new lens sample. We focus on the two key quantities studied in Section 7: the density profile slope $\gamma^{\prime}$, and the dark matter fraction $f_{\mathrm{DM}}$.

The low redshift reference measurements of $\gamma^{\prime}$ and $f_{\mathrm{DM}}$ come from the SLACS analysis, which found density profiles very close to isothermal inside one effective radius in a sample of 63 SLACS strong-lens early-type galaxies: $\left\langle\gamma_{\text {SLACS }}^{\prime}\right\rangle=2.078 \pm 0.027$, with a scatter of 0.16 (Auger et al. 2010). Likewise Auger et al. (2009) inferred a mean $f_{\mathrm{DM}}$ within $R_{\mathrm{eff}} / 2$ for 85 SLACS lenses of 0.3 with a scatter of 0.2 , using a Salpeter IMF. These results are broadly consistent with our findings for SL2S systems. We can therefore anticipate only a mild cosmic evolution of these quantities.

In Figure 13, we show how the density profile slope $\gamma^{\prime}$ varies with redshift, using the SL2S, SLACS and LSD samples together to cover the redshift range 0.05 to 1 . We quantify this statement by fitting the $\gamma^{\prime}\left(z_{\mathrm{d}}\right)$ data with a linear relation in the mean slope, still including Gaussian scatter about that relation:

$$
\left\langle\gamma^{\prime}\right\rangle\left(z_{\mathrm{d}}\right)=\left\langle\gamma_{0}^{\prime}\right\rangle+\frac{\partial\left\langle\gamma^{\prime}\right\rangle}{\partial z_{\mathrm{d}}} z_{\mathrm{d}} \pm S_{\gamma^{\prime}} .
$$

For the SL2S data alone, we find $\left\langle\gamma_{0}^{\prime}\right\rangle=2.22_{-0.21}^{+0.17}$, $\partial\left\langle\gamma^{\prime}\right\rangle / \partial z_{\mathrm{d}}=-0.16_{-0.51}^{+0.48}$ for the gradient and, in this 


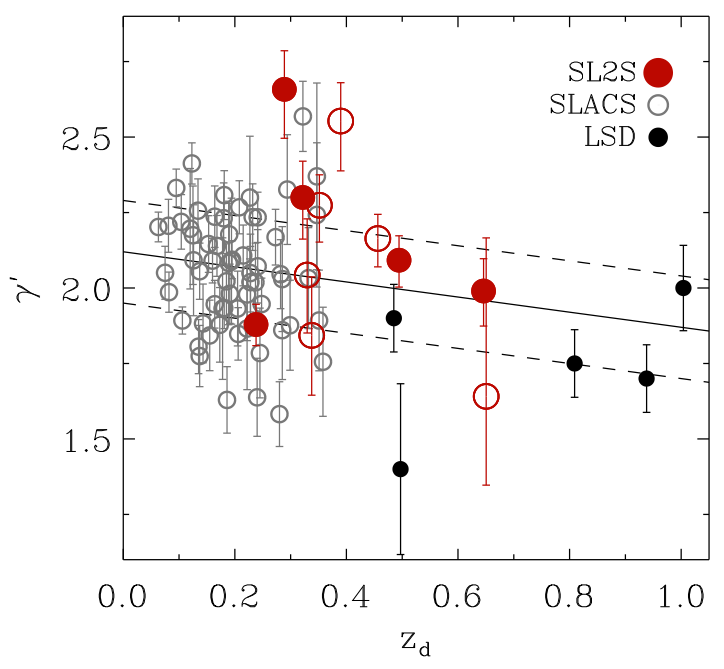

FIG. 13. - Cosmic evolution of total mass density slope, $\gamma^{\prime}$. The SLACS and LSD values were taken from: (Auger et al. 2010) and (Treu \& Koopmans 2002; Koopmans \& Treu 2003; Treu \& Koopmans 2004), respectively. The error bars show the $16^{\text {th }}$ and $84^{\text {th }}$ percentiles. The best fit to the data is shown by the solid line and the scatter is shown by the dashed lines.

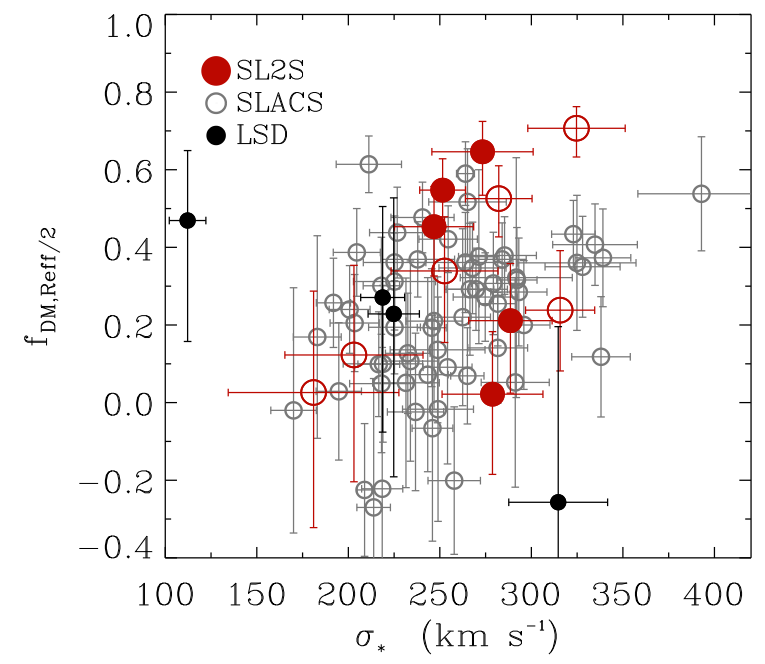

FIG. 14. - The dark matter fraction within $R_{\text {eff }} / 2$ as a function of measured velocity dispersion. The error bars show the $16^{\text {th }}$ and $84^{\text {th }}$ percentiles. For all samples, the velocity dispersion was normalized to a standard aperture, $R_{\text {eff }} / 2$.

evolving $\gamma^{\prime}$ case, the scatter is $S_{\gamma^{\prime}}=0.23_{-0.06}^{+0.09}$. When we include the SLACS and LSD data points, we find $\left\langle\gamma_{0}^{\prime}\right\rangle=$ $2.12_{-0.04}^{+0.03}, \partial\left\langle\gamma^{\prime}\right\rangle / \partial z_{\mathrm{d}}=-0.25_{-0.12}^{+0.10}$, and $S_{\gamma^{\prime}}=0.17_{-0.02}^{+0.02}$.

These results are inconsistent with no evolution in the total density profile slope of massive lens galaxies since $z \simeq 1$ : the probability of the linear gradient in $\left\langle\gamma^{\prime}\right\rangle$ being positive is just $2 \%$. The lens data suggest (at approximately the $2-\sigma$ level) that the mean total density profile of massive galaxies has become slightly steeper over cosmic time.

Figure 14 shows the dark matter fraction as a function of the measured stellar velocity dispersion, $\sigma_{\mathrm{e} 2}$ for the SL2S, SLACS and LSD samples. Together, these samples cover a large rage in stellar velocity dispersion, however, no significant trend with central dark matter fraction was

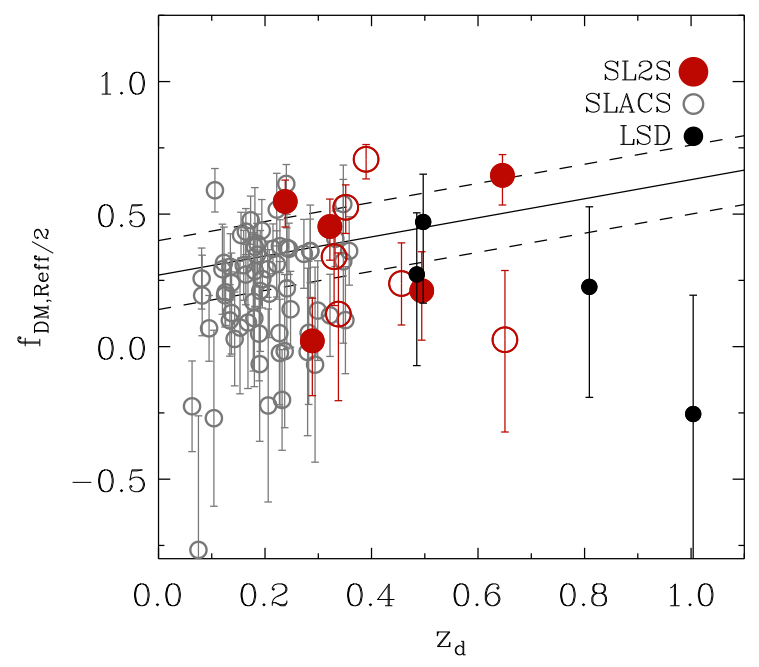

FIG. 15. - Evolution of dark matter fraction with redshift for a Salpeter IMF. The error bars show the $16^{\text {th }}$ and $84^{\text {th }}$ percentiles. The solid and dashed lines show the best fit to the data and the scatter, respectively.

found. In Figure 15, we show how the dark matter fraction varies with redshift, using the SL2S, SLACS and LSD samples together to cover the redshift range 0.05 to 1.0, and focusing on the Salpeter IMF. (The Chabrier IMF assignment only affects the overall normalization of the stellar masses and not their evolution.) Only 4 of the 5 LSD lenses were included, since photometry in multiple bands was required to infer stellar masses (given in Section 4.2). We find that the mean dark matter fraction has not evolved strongly with cosmic time, however, there is marginal evidence for some change in the population. Again, we quantify this statement by fitting the $f_{\mathrm{DM}}\left(z_{\mathrm{d}}\right)$ data with a linear relation in the mean slope, still including Gaussian scatter about that relation:

$$
\left\langle f_{\mathrm{DM}}\right\rangle\left(z_{\mathrm{d}}\right)=\left\langle f_{\mathrm{DM}, 0}\right\rangle+\frac{\partial\left\langle f_{\mathrm{DM}}\right\rangle}{\partial z_{\mathrm{d}}} z_{\mathrm{d}} \pm S_{f_{\mathrm{DM}}} .
$$

For the SL2S data alone, we find $\left\langle f_{\mathrm{DM}, 0}\right\rangle=0.38_{-0.50}^{+0.12}$, $\partial\left\langle f_{\mathrm{DM}}\right\rangle / \partial z_{\mathrm{d}}=0.07_{-0.36}^{+0.36}$ for the gradient and $S_{f_{\mathrm{DM}}}=$ $0.18_{-0.07}^{+0.08}$ for the scatter. Including the SLACS and LSD data points, we find $\left\langle f_{\mathrm{DM}, 0}\right\rangle=0.27_{-0.06}^{+0.06}, \partial\left\langle f_{\mathrm{DM}}\right\rangle / \partial z_{\mathrm{d}}=$ $0.36_{-0.24}^{+0.18}$, and $S_{f_{\mathrm{DM}}}=0.13_{-0.02}^{+0.02}$. The probability of the linear gradient in $\left\langle f_{\mathrm{DM}}\right\rangle$ being positive is $98 \%$ : the lens data suggest (again at approximately the 2- $\sigma$ level) that the mean projected dark matter fraction in massive galaxies, within half their effective radius, has decreased slightly over cosmic time.

\section{DISCUSSION}

By combining the inferred total mass density slopes of the SL2S, SLACS and LSD samples, we found a tantalizing suggestion that $\gamma^{\prime}$ has become slightly steeper over cosmic time.

Before interpreting this result, and its implications for our understanding of the formation and evolution of early-type galaxies, it is important to address a potential source of systematic error. As shown in Figure 16, the ratio of $R_{\text {Ein }} / R_{\text {eff }}$ increases with redshift, mainly because the physical size of $R_{\text {Ein }}$ increases with redshift (as discussed in Section 6.2), but also because of the increasing 


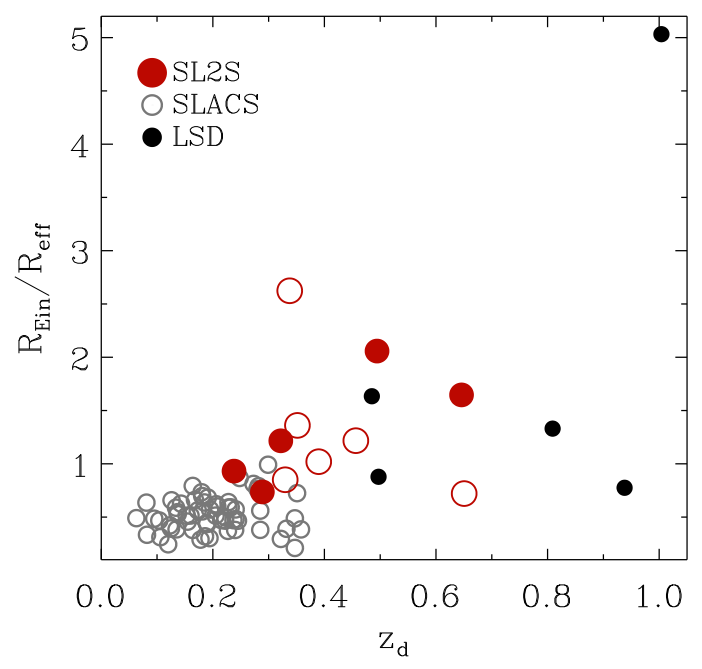

FIG. 16. - Evolution of $R_{\text {Ein }} / R_{\text {eff }}$ with redshift. The ratio of $R_{\text {Ein }} / R_{\text {eff }}$ tends to increase with redshift.

source redshift. Since the slope of the total mass density is determined by measuring the total mass at two different radii, one of which is the Einstein radius, SL2S lenses sample $\gamma^{\prime}$ at larger radii than SLACS lenses. Therefore, a trend in $R_{\text {Ein }} / R_{\text {eff }}$ with redshift could mimic the inferred evolution of $\gamma^{\prime}$. However, for this effect to mimic the evolution of $\gamma^{\prime}$, the density profile would have to become shallower and then steeper with increasing radius, which is unlikely given the total mass density profile of local early-type galaxies. We also note that $\gamma^{\prime}$ and $f_{\mathrm{DM}}$ vary differently with cosmic time, which is unexpected if the evolution being mimicked by a trend in $R_{\text {Ein }} / R_{\text {eff }}$ with redshift. As discussed in Sections 7.4 and 7.5, Figures 10 and 12 show that both $\gamma^{\prime}$ and $f_{\mathrm{DM}}$ show a negligible trend with $R_{\text {Ein }} / R_{\text {eff }}$, suggesting that the evolution of $\gamma^{\prime}$ is not an artifact due to a dependence on $R_{\text {Ein }} / R_{\text {eff }}$.

For these reasons we conclude that true evolution of the average mass density profile is the most likely interpretation of our findings, although additional measurements of $\gamma^{\prime}$ at different radii are needed to conclusively rule out alternate interpretations.

It is important to stress that both the mean value and evolution of $\gamma^{\prime}$ are completely consistent with previous results, $\left\langle\gamma_{0, \mathrm{SLACS}+\mathrm{LSD}}^{\prime}\right\rangle=2.10 \pm 0.07$ and $\partial\left\langle\gamma^{\prime}\right\rangle / \partial z_{\mathrm{d}}=$ $-0.23 \pm 0.16$ (Koopmans et al. 2006). It is only by virtue of our larger sample that we have been able to reduce the error bars and find marginal evidence for evolution. If this evidence for evolution is confirmed by larger samples, it would indicate that growth of massive galaxies since $z \sim 1$ has not occurred through dry (dissipationless) mergers alone, since they preserve $\gamma^{\prime}$ (see e.g. Nipoti et al. 2009b). In dissipative merging events, $\gamma^{\prime}$ increases as a result of baryons cooling and sinking towards the center. Therefore, dissipative processes would be required to contribute, at least partially, to the evolution of early-type galaxies since $z \sim 1$. Although they cannot be the dominant process, because of tight limits on recent star formation in massive early-type galaxies since $z \sim 1$ (e.g., Treu et al. 2005), our result seems to suggest that they cannot be completely neglected either. This is consistent with evidence for a "frosting" of recent stars found in detailed studies of the stellar populations of massive galaxies (e.g., Trager et al. 2000).

Another notable feature of the SL2S $\gamma^{\prime}$ distribution is its large intrinsic scatter. The intrinsic scatter in the SLACS sample is just 0.16 \pm 0.02 (Auger et al. 2010), compared to $0.25_{-0.07}^{+0.10}$ for SL2S. From the LSD lenses, Treu \& Koopmans (2004) also found that the intrinsic scatter in $\gamma^{\prime}$ was larger at $z=1$. This may indicate an overall trend toward more complete dynamical relaxation over Gyr timescales, and perhaps a reduced contribution of external convergence, due to line of sight structure in nearby systems, where the Einstein radii are smaller. Again, a larger sample size is required to confirm this interpretation. Finally, we find that the dark matter fraction within half of the effective radius has decreased slightly with cosmic time. This is again consistent with some contribution from dissipational processes in early-type galaxy formation and evolution, where baryons move to the central region as they cool. However, it is important to keep in mind residual uncertainties in estimating the stellar mass, which may be redshift dependent, since the average age of the stellar populations is a function of cosmic time. Cosmic evolution of the IMF would be another source of ambiguity in interpreting these results (van Dokkum 2008; Treu et al. 2010).

To conclude, we have developed a new method to estimate source redshifts, in the absence of spectroscopic measurements. The method is completely general, does not require accurate multiband photometry of the sources, and allows us to infer $\gamma^{\prime}$ and $f_{\mathrm{DM}}$ with errors that are just 2-3 times as large as if we had spectroscopic redshifts. Although spectroscopic redshifts are in general preferable, this may be a good strategy for exploiting future surveys of thousands of strong lenses, where wholesale spectroscopy of the complete sample may not be practical.

\section{SUMMARY AND CONCLUSIONS}

New spectroscopic measurements with deflector redshifts and velocity dispersions were presented for 11 lenses. These spectroscopic measurements were combined with lens models and photometry, described in Paper I, to infer the total density slope and dark matter fraction of each of the 11 SL2S galaxies in the final sample.

The main results are summarized below:

1. The SL2S sample has a median deflector redshift, $z_{\mathrm{d}}=0.494$, source redshift, $z_{\mathrm{s}}=1.199$ and velocity dispersion, $\sigma_{\mathrm{e} 2}=273 \mathrm{~km} \mathrm{~s}^{-1}$.

2. The SL2S, SLACS and LSD lenses are the same types of galaxies, however, the physical size of $R_{\text {Ein }}$ is generally larger for higher redshift deflectors.

3. We developed a new method to estimate the source redshift probability distribution function for lenses with no spectroscopic $z_{\mathrm{s}}$. This lack of accurate source redshift produces uncertainties on $\gamma^{\prime}$ and $f_{\mathrm{DM}}$ that are only a factor 2-3 greater. Uncertainties would eventually further decrease with better multiband source photometry, and this would allow large samples of high redshift lenses to be analyzed in the next generation of cosmological sur- 
veys, where spectroscopy of all the systems may not be affordable.

4. The average total density slope measured from the SL2S sample alone is: $\left\langle\gamma_{0}^{\prime}\right\rangle=2.16_{-0.09}^{+0.09}$, with a scatter of $0.25_{-0.07}^{+0.10}$.

5. Combining the SL2S $\gamma^{\prime}$ measurements with previous analyses from SLACS and LSD, we find, $\left\langle\gamma_{0}^{\prime}\right\rangle=2.12_{-0.04}^{+0.03}, \partial\left\langle\gamma^{\prime}\right\rangle / \partial z_{\mathrm{d}}=-0.25_{-0.12}^{+0.10}$, and $S_{\gamma^{\prime}}=$ $0.17_{-0.02}^{+0.02}$. This suggests (at approximately the $2-\sigma$ level) that the mean total density profile of massive galaxies has become slightly steeper over cosmic time.

6. Stellar masses were estimated using CFHT photometry, and enabled us to disentangle the total and stellar masses.

7. From this we inferred the dark matter fraction within half the effective radius: $\left\langle f_{\mathrm{DM}, 0}\right\rangle=$ $0.38_{-0.50}^{+0.12}$, with a scatter $S_{f_{\mathrm{DM}}}=0.18_{-0.07}^{+0.08}$ for the SL2S sample, using a Salpeter IMF.

8. The combined dark matter fractions from all three samples suggest that the dark matter fraction within $R_{\text {eff }} / 2$ has decreased slightly since $z \sim 1$. We find the mean dark matter fraction within $R_{\text {eff }} / 2$, gradient of evolution over cosmic time and scatter to be: $\left\langle f_{\mathrm{DM}, 0}\right\rangle=0.27_{-0.06}^{+0.06}, \partial\left\langle f_{\mathrm{DM}}\right\rangle / \partial z_{\mathrm{d}}=$ $0.36_{-0.24}^{+0.18}$, and $S_{f_{\mathrm{DM}}}=0.13_{-0.02}^{+0.02}$, respectively.

We thank our friends of the SLACS and SL2S collaborations for many useful and insightful discussions over the course of the past years.

AJR acknowledges the support of an Australian Postgraduate Award. RG and FB acknowledge support from the Centre National des Etudes Spatiales (CNES). PJM was given support by the TABASGO and Kavli foundations in the form of two research fellowships. TT acknowledges support from the NSF through CAREER award NSF-0642621, and from the Packard Foundation through a Packard Research Fellowship. Based on observations obtained with MegaPrime/MegaCam, a joint project of CFHT and CEA/DAPNIA, at the CanadaFrance-Hawaii Telescope (CFHT) which is operated by the National Research Council (NRC) of Canada, the Institut National des Sciences de l'Univers of the Centre National de la Recherche Scientifique (CNRS) of France, and the University of Hawaii. This work is based in part on data products produced at TERAPIX and the Canadian Astronomy Data Centre as part of the CanadaFrance-Hawaii Telescope Legacy Survey, a collaborative project of NRC and CNRS. This research is supported by NASA through Hubble Space Telescope programs GO10876, GO-11289, GO-11588 and in part by the National Science Foundation under Grant No. PHY99-07949, and is based on observations made with the NASA/ESA Hubble Space Telescope and obtained at the Space Telescope Science Institute, which is operated by the Association of Universities for Research in Astronomy, Inc., under NASA contract NAS 5-26555, and at the W.M. Keck Observatory, which is operated as a scientific partnership among the California Institute of Technology, the University of California and the National Aeronautics and Space Administration. The Observatory was made possible by the generous financial support of the W.M. Keck Foundation. The authors wish to recognize and acknowledge the very significant cultural role and reverence that the summit of Mauna Kea has always had within the indigenous Hawaiian community. We are most fortunate to have the opportunity to conduct observations from this mountain.

\section{REFERENCES}

Auger, M. W., Treu, T., Bolton, A. S., Gavazzi, R., Koopmans, L. V. E., Marshall, P. J., Bundy, K., \& Moustakas, L. A. 2009, ApJ, 705, 1099

Auger, M. W., Treu, T., Bolton, A. S., Gavazzi, R., Koopmans, L. V. E., Marshall, P. J., \& Burles, S. 2010, ApJ

Barnabe, M., Auger, M. W., Treu, T., Koopmans, L., Bolton, A. S., Czoske, O., \& Gavazzi, R. 2010, MNRAS, 955

Barnabè, M., Czoske, O., Koopmans, L. V. E., Treu, T., Bolton, A. S., \& Gavazzi, R. 2009, MNRAS, 399, 21

Bernardi, M., Sheth, R. K., Nichol, R. C., Schneider, D. P., \& Brinkmann, J. 2005, AJ, 129, 61

Bertin, G., \& Stiavelli, M. 1993, Reports on Progress in Physics, 56,493

Bolton, A. S., Burles, S., Koopmans, L. V. E., Treu, T., Gavazzi, R., Moustakas, L. A., Wayth, R., \& Schlegel, D. J. 2008a, ApJ, 682,964

Bolton, A. S., Burles, S., Koopmans, L. V. E., Treu, T., \& Moustakas, L. A. 2006, ApJ, 638, 703

Bolton, A. S., Treu, T., Koopmans, L. V. E., Gavazzi, R., Moustakas, L. A., Burles, S., Schlegel, D. J., \& Wayth, R. 2008b, ApJ, 684, 248

Bruzual, G., \& Charlot, S. 2003, MNRAS, 344, 1000

Bundy, K., Ellis, R. S., \& Conselice, C. J. 2005, ApJ, 625, 621

Bundy, K., Treu, T., \& Ellis, R. S. 2007, ApJ, 665, L5

Cabanac, R. A., Alard, C., Dantel-Fort, M., Fort, B., Gavazzi, R., Gomez, P., Kneib, J. P., Le Fèvre, O., Mellier, Y., Pello, R., Soucail, G., Sygnet, J. F., \& Valls-Gabaud, D. 2007, A\&A, 461, 813
Cappellari, M., Bacon, R., Bureau, M., Damen, M. C., Davies, R. L., de Zeeuw, P. T., Emsellem, E., Falcón-Barroso, J., Krajnović, D., Kuntschner, H., McDermid, R. M., Peletier, R. F., Sarzi, M., van den Bosch, R. C. E., \& van de Ven, G. 2006, MNRAS, 366, 1126

Cappellari, M., di Serego Alighieri, S., Cimatti, A., Daddi, E., Renzini, A., Kurk, J. D., Cassata, P., Dickinson, M., Franceschini, A., Mignoli, M., Pozzetti, L., Rodighiero, G., Rosati, P., \& Zamorani, G. 2009, ApJ, 704, L34

Cardone, V. F., \& Tortora, C. 2010, ArXiv e-prints

Cardone, V. F., Tortora, C., Molinaro, R., \& Salzano, V. 2009, A\&A, 504, 769

Cassata, P., Giavalisco, M., Guo, Y., Ferguson, H., Koekemoer, A. M., Renzini, A., Fontana, A., Salimbeni, S., Dickinson, M., Casertano, S., Conselice, C. J., Grogin, N., Lotz, J. M., Papovich, C., Lucas, R. A., Straughn, A., Gardner, J. P., \& Moustakas, L. 2010, ApJ, 714, L79

Chabrier, G. 2003, PASP, 115, 763

Cimatti, A., Daddi, E., \& Renzini, A. 2006, A\&A, 453, L29

Ciotti, L. 2009, Nature, 460, 333

Ciotti, L., Lanzoni, B., \& Volonteri, M. 2007, ApJ, 658, 65

Ciotti, L., Ostriker, J. P., \& Proga, D. 2009, ApJ, 699, 89

Croton, D. J., Springel, V., White, S. D. M., De Lucia, G., Frenk, C. S., Gao, L., Jenkins, A., Kauffmann, G., Navarro, J. F., \& Yoshida, N. 2006, MNRAS, 365, 11 
Daddi, E., Renzini, A., Pirzkal, N., Cimatti, A., Malhotra, S., Stiavelli, M., Xu, C., Pasquali, A., Rhoads, J. E., Brusa, M., di Serego Alighieri, S., Ferguson, H. C., Koekemoer, A. M., Moustakas, L. A., Panagia, N., \& Windhorst, R. A. 2005, ApJ, 626,680

de Vaucouleurs, G. 1948, Annales d'Astrophysique, 11, 247

Dehnen, W. 2005, MNRAS, 360, 892

di Serego Alighieri, S., Vernet, J., Cimatti, A., Lanzoni, B., Cassata, P., Ciotti, L., Daddi, E., Mignoli, M., Pignatelli, E., Pozzetti, L., Renzini, A., Rettura, A., \& Zamorani, G. 2005, A\&A, 442, 125

Faure, C., Kneib, J.-P., Covone, G., Tasca, L., Leauthaud, A., Capak, P., Jahnke, K., Smolcic, V., de la Torre, S., Ellis, R., Finoguenov, A., Koekemoer, A., Le Fevre, O., Massey, R., Mellier, Y., Refregier, A., Rhodes, J., Scoville, N., Schinnerer, E., Taylor, J., Van Waerbeke, L., \& Walcher, J. 2008, ApJS, 176,19

Gavazzi, R., Treu, T., Rhodes, J. D., Koopmans, L. V. E., Bolton, A. S., Burles, S., Massey, R. J., \& Moustakas, L. A. 2007, ApJ, 667,176

Graves, G. J., Faber, S. M., \& Schiavon, R. P. 2009, ApJ, 693, 486

Grillo, C., Gobat, R., Rosati, P., \& Lombardi, M. 2008, A\&A, 477, L25

Hopkins, P. F., Bundy, K., Hernquist, L., Wuyts, S., \& Cox, T. J. 2010, MNRAS, 401, 1099

Hyde, J. B., \& Bernardi, M. 2009, MNRAS, 396, 1171

Jiang, G., \& Kochanek, C. S. 2007, ApJ, 671, 1568

Jørgensen, I., Franx, M., \& Kjaergaard, P. 1995, MNRAS, 276, 1341

Juneau, S., Glazebrook, K., Crampton, D., McCarthy, P. J., Savaglio, S., Abraham, R., Carlberg, R. G., Chen, H., Le Borgne, D., Marzke, R. O., Roth, K., Jørgensen, I., Hook, I., \& Murowinski, R. 2005, ApJ, 619, L135

Kazantzidis, S., Zentner, A. R., \& Kravtsov, A. V. 2006, ApJ, 641, 647

Khochfar, S., \& Silk, J. 2006, ApJ, 648, L21

Kochanek, C. S. 1994, ApJ, 436, 56

Koopmans, L. V. E., Bolton, A., Treu, T., Czoske, O., Auger, M. W., Barnabè, M., Vegetti, S., Gavazzi, R., Moustakas, L. A., \& Burles, S. 2009, ApJ, 703, L51

Koopmans, L. V. E., \& Treu, T. 2002, ApJ, 568, L5

-. 2003, ApJ, 583, 606

Koopmans, L. V. E., Treu, T., Bolton, A. S., Burles, S., \& Moustakas, L. A. 2006, ApJ, 649, 599

Kormann, R., Schneider, P., \& Bartelmann, M. 1994, A\&A, 284, 285

Lackner, C. N., \& Ostriker, J. P. 2010, ApJ, 712, 88

Lagattuta, D. J., Fassnacht, C. D., Auger, M. W., Marshall, P. J. Bradač, M., Treu, T., Gavazzi, R., Schrabback, T., Faure, C., \& Anguita, T. 2010, ApJ, 716, 1579

Leauthaud, A., et al. 2007, ApJS, 172, 219

Lewis, A., \& Bridle, S. 2002, Phys. Rev. D, 66, 103511

Mancini, C., Daddi, E., Renzini, A., Salmi, F., McCracken, H. J., Cimatti, A., Onodera, M., Salvato, M., Koekemoer, A. M., Aussel, H., Floc'h, E. L., Willott, C., \& Capak, P. 2010, MNRAS, 401, 933

Marshall, P. J., Treu, T., Melbourne, J., Gavazzi, R., Bundy, K., Ammons, S. M., Bolton, A. S., Burles, S., Larkin, J. E., Le Mignant, D., Koo, D. C., Koopmans, L. V. E., Max, C. E., Moustakas, L. A., Steinbring, E., \& Wright, S. A. 2007, ApJ, 671,1196

Merritt, D. 1999, PASP, 111, 129

Meza, A., Navarro, J. F., Steinmetz, M., \& Eke, V. R. 2003, ApJ, 590,619

Miralda-Escude, J. 1995, ApJ, 438, 514

Naab, T., Johansson, P. H., \& Ostriker, J. P. 2009, ApJ, 699, L178

Naab, T., Johansson, P. H., Ostriker, J. P., \& Efstathiou, G. 2007, ApJ, 658, 710
Natarajan, P., \& Kneib, J.-P. 1996, MNRAS, 283, 1031

Navarro, J. F., Ludlow, A., Springel, V., Wang, J., Vogelsberger, M., White, S. D. M., Jenkins, A., Frenk, C. S., \& Helmi, A. 2010, MNRAS, 402, 21

Newman, A. B., Ellis, R. S., Treu, T., \& Bundy, K. 2010, ApJ, 717, L103

Nipoti, C., Treu, T., Auger, M. W., \& Bolton, A. S. 2009a, ApJ, 706, L86

Nipoti, C., Treu, T., \& Bolton, A. S. 2009b, ApJ, 703, 1531

Oñorbe, J., Domínguez-Tenreiro, R., Sáiz, A., \& Serna, A. 2007, MNRAS, 376, 39

Ohyama, Y., Hamana, T., Kashikawa, N., Chiba, M., Futamase, T., Iye, M., Kawabata, K. S., Aoki, K., Sasaki, T., Kosugi, G., \& Takata, T. 2002, AJ, 123, 2903

Renzini, A. 2006, ARA\&A, 44, 141

Robertson, B., Cox, T. J., Hernquist, L., Franx, M., Hopkins, P. F., Martini, P., \& Springel, V. 2006, ApJ, 641, 21

Salpeter, E. E. 1955, ApJ, 121, 161

Saracco, P., Longhetti, M., \& Andreon, S. 2009, MNRAS, 392, 718

Schlegel, D. J., Finkbeiner, D. P., \& Davis, M. 1998, ApJ, 500, 525

Sheth, R. K., Bernardi, M., Schechter, P. L., Burles, S., Eisenstein, D. J., Finkbeiner, D. P., Frieman, J., Lupton, R. H., Schlegel, D. J., Subbarao, M., Shimasaku, K., Bahcall, N. A., Brinkmann, J., \& Ivezić, Ž. 2003, ApJ, 594, 225

Suyu, S. H., Marshall, P. J., Auger, M. W., Hilbert, S., Blandford, R. D., Koopmans, L. V. E., Fassnacht, C. D., \& Treu, T. 2010, ApJ, 711, 201

Suyu, S. H., Marshall, P. J., Blandford, R. D., Fassnacht, C. D., Koopmans, L. V. E., McKean, J. P., \& Treu, T. 2009, ApJ, 691, 277

Thomas, D., Maraston, C., Bender, R., \& Mendes de Oliveira, C. 2005, ApJ, 621, 673

Trager, S. C., Faber, S. M., Worthey, G., \& González, J. J. 2000, AJ, 120, 165

Treu, T., Auger, M. W., Koopmans, L. V. E., Gavazzi, R. Marshall, P. J., \& Bolton, A. S. 2010, ApJ, 709, 1195

Treu, T., Ellis, R. S., Liao, T. X., van Dokkum, P. G., Tozzi, P., Coil, A., Newman, J., Cooper, M. C., \& Davis, M. 2005, ApJ, 633,174

Treu, T., Gavazzi, R., Gorecki, A., Marshall, P. J., Koopmans, L. V. E., Bolton, A. S., Moustakas, L. A., \& Burles, S. 2009, ApJ, 690, 670

Treu, T., Koopmans, L. V., Bolton, A. S., Burles, S., \& Moustakas, L. A. 2006, ApJ, 640, 662

Treu, T., \& Koopmans, L. V. E. 2002, ApJ, 575, 87

-. 2004, ApJ, 611, 739

Treu, T., Stiavelli, M., Walker, A. R., Williams, R. E., Baum, S. A., Bernstein, G., Blacker, B. S., Carollo, C. M., Casertano, S., Dickinson, M. E., De Mello, D. F., Ferguson, H. C., Fruchter, A. S., Lucas, R. A., MacKenty, J., Madau, P., \& Postman, M. 1998, A\&A, 340, L10

van Albada, T. S., \& Sancisi, R. 1986, Royal Society of London Philosophical Transactions Series A, 320, 447

van der Marel, R. P. 1994, MNRAS, 270, 271

van der Wel, A., Bell, E. F., van den Bosch, F. C., Gallazzi, A., \& Rix, H.-W. 2009, ApJ, 698, 1232

van der Wel, A., Franx, M., van Dokkum, P. G., Rix, H.-W., Illingworth, G. D., \& Rosati, P. 2005, ApJ, 631, 145

van Dokkum, P. G. 2008, ApJ, 674, 29

van Dokkum, P. G., Franx, M., Kriek, M., Holden, B., Illingworth, G. D., Magee, D., Bouwens, R., Marchesini, D., Quadri, R., Rudnick, G., Taylor, E. N., \& Toft, S. 2008, ApJ, 677, L5

Wucknitz, O., Biggs, A. D., \& Browne, I. W. A. 2004, MNRAS, 349,14 
TABLE 2

Measured SL2S galaXY-SCALE Lens PRoperties

\begin{tabular}{|c|c|c|c|c|c|c|c|c|c|c|c|c|c|c|c|c|}
\hline Name & $z_{\mathrm{d}}$ & $z_{\mathrm{s}}$ & $z_{\mathrm{s}, \mathrm{pdf}}$ & $\begin{array}{c}\sigma \\
\left(\mathrm{km} \mathrm{s}^{-1}\right)\end{array}$ & $\begin{array}{c}\mathrm{S} / \mathrm{N} \\
\left(\AA^{-1}\right)\end{array}$ & $\begin{array}{c}R_{\operatorname{Ein}} \\
(\operatorname{arcsec})\end{array}$ & $q_{\text {mass }}$ & $\begin{array}{c}R_{\mathrm{eff}} \\
(\operatorname{arcsec})\end{array}$ & $q_{*}$ & $m_{\mathrm{u}}$ & $m_{\mathrm{g}}$ & $m_{\mathrm{r}}$ & $m_{\mathrm{i}}$ & $m_{\mathrm{z}}$ & Flag & Run \\
\hline J021411-040502 & 0.6080 & - & $1.57_{-0.35}^{+0.50}$ & - & 6.1 & 0.918 & 0.33 & 0.94 & 0.89 & 22.46 & 21.08 & 19.57 & 18.78 & 19.41 & HST & $2 \mathrm{~b}$ \\
\hline J021737-051329 & 0.6458 & 1.847 & $1.74_{-0.37}^{+0.53}$ & $257 \pm 26$ & 14.5 & 1.268 & 0.91 & 0.77 & 0.89 & 21.57 & 20.45 & 19.51 & 19.47 & 19.16 & HST & $2 \mathrm{~b}, 4 \mathrm{c}$ \\
\hline J021902-082934 & 0.3898 & - & $1.30_{-0.43}^{+0.65}$ & $305 \pm 25$ & 19.7 & 0.918 & 0.53 & 0.90 & 0.72 & 22.50 & 20.77 & 20.14 & 19.33 & 18.70 & CFHT & $3 \mathrm{a}$ \\
\hline J022056-063934 & 0.3297 & - & $1.47_{-0.49}^{+0.68}$ & $242 \pm 28$ & 25.7 & 1.250 & 0.73 & 1.47 & 0.57 & 21.09 & 20.12 & 18.30 & 18.35 & 17.62 & CFHT & $3 b$ \\
\hline J022511-045433 & 0.2380 & 1.1988 & $1.25_{-0.39}^{+0.55}$ & $241 \pm 12$ & 53.0 & 1.770 & 0.58 & 1.90 & 0.75 & 19.37 & 17.91 & 17.39 & 16.84 & 16.74 & HST & 5 \\
\hline J022610-042011 & 0.4943 & 1.232 & $1.54_{-0.40}^{+0.60}$ & $266 \pm 21$ & 16.4 & 1.153 & 0.92 & 0.56 & 0.94 & 22.04 & 20.47 & 19.66 & 18.78 & 18.58 & HST & $4 a$ \\
\hline J022648-040610 & 0.7663 & - & $2.18_{-0.47}^{+0.67}$ & - & 9.4 & 1.306 & 0.82 & 1.20 & 0.37 & 23.34 & 22.15 & 21.40 & 20.00 & 19.60 & HST & $2 \mathrm{a}$ \\
\hline J022648-090421 & 0.4563 & - & $1.98_{-0.57}^{+0.78}$ & $301 \pm 18$ & 30.8 & 1.582 & 0.83 & 1.30 & 0.80 & 22.47 & 20.06 & 18.14 & 18.32 & 17.73 & CFHT & $4 a$ \\
\hline J023251-040823 & 0.3516 & - & $1.52_{-0.53}^{+0.74}$ & $264 \pm 17$ & 22.6 & 1.102 & 0.80 & 0.81 & 0.67 & 21.87 & 20.13 & 18.41 & 18.58 & 18.52 & HST & $3 a$ \\
\hline $\mathrm{J} 140123+555705$ & 0.5263 & - & $1.62_{-0.42}^{+0.58}$ & - & 10.0 & 1.186 & 0.49 & 0.76 & 0.73 & 22.77 & 21.35 & 20.15 & 19.14 & 18.58 & HST & 1 \\
\hline $\mathrm{J} 140614+520252$ & 0.4797 & - & - & - & 8.5 & - & - & 2.15 & 0.49 & 22.05 & 20.06 & 18.47 & 18.29 & 17.93 & CFHT & 1 \\
\hline $\mathrm{J} 141137+565119$ & 0.3218 & 1.420 & $1.37_{-0.52}^{+0.77}$ & $228 \pm 20$ & 34.0 & 0.924 & 0.90 & 0.76 & 0.74 & 21.08 & 19.75 & 18.59 & 18.49 & 18.32 & HST & 6 \\
\hline $\mathrm{J} 220629+005728$ & 0.7044 & - & - & - & 10.4 & - & - & 2.25 & 0.99 & 23.11 & 21.47 & 20.64 & 20.59 & 19.04 & CFHT & $3 a$ \\
\hline $\mathrm{J} 221326-000946$ & 0.3378 & - & $1.30_{-0.44}^{+0.63}$ & $183 \pm 34$ & 18.9 & 1.076 & 0.19 & 0.41 & 0.30 & 23.93 & 22.31 & 20.10 & 20.00 & 19.49 & HST & 5 \\
\hline $\mathrm{J} 221407-180712$ & 0.6505 & - & $1.12_{-0.22}^{+0.46}$ & $167 \pm 43$ & 8.3 & 0.411 & 0.69 & 0.57 & 0.65 & 23.91 & 21.96 & 21.04 & 20.43 & 19.79 & HST & $3 b$ \\
\hline J221606-175131 & 0.8602 & - & - & $282 \pm 44$ & 13.5 & - & - & 0.93 & 0.87 & 23.35 & 22.39 & 21.36 & 20.68 & 19.72 & CFHT & $3 a$ \\
\hline $\mathrm{J} 221929-001743$ & 0.2888 & 1.0232 & $0.91_{-0.32}^{+0.54}$ & $263 \pm 26$ & 44.4 & 0.736 & 0.75 & 1.00 & 0.75 & 20.39 & 18.38 & 16.78 & 17.78 & 17.61 & CFHT & $4 \mathrm{a}, 4 \mathrm{~b}$ \\
\hline
\end{tabular}

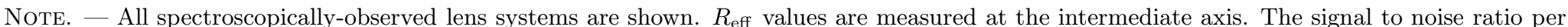
pixel was calculated over the rest wavelength range 4000-5000A. Typical uncertainties on $R_{\text {Ein }}$ and $R_{\text {eff }}$ are $5 \%$ and $10 \%$, respectively. The column, Flag, indicates whether HST or CFHT data was used to measure $R_{\text {Ein }}$ and $R_{\text {eff }}$. 
TABLE 3

INFERRED SL2S GALAXY-SCALE LENS PROPERTIES

\begin{tabular}{|c|c|c|c|c|c|c|c|c|}
\hline Name & $\begin{array}{l}R_{\operatorname{Ein}} \\
(\mathrm{kpc})\end{array}$ & $\begin{array}{c}\sigma_{\mathrm{e} 2} \\
\left(\mathrm{~km} \mathrm{~s}^{-1}\right)\end{array}$ & $\begin{array}{c}\sigma_{\mathrm{SIE}} \\
\left(\mathrm{km} \mathrm{s}^{-1}\right)\end{array}$ & $\begin{array}{c}M_{*} \\
\left(10^{11} M_{\odot}\right)\end{array}$ & $\begin{array}{c}M_{\operatorname{Ein}} \\
\left(10^{11} M_{\odot}\right)\end{array}$ & $\gamma^{\prime}$ & $f_{\mathrm{DM}, R_{\text {eff }} 2}^{\mathrm{Salp}}$ & $f_{\mathrm{DM}, R_{\text {eff }} 2}^{\mathrm{Chab}}$ \\
\hline J021737-051329 & 8.76 & $273 \pm 27$ & 289 & $1.77_{-0.37}^{+0.53}$ & 5.35 & $1.99_{-0.12}^{+0.11}$ & $0.65_{-0.08}^{+0.11}$ & $0.80_{-0.04}^{+0.11}$ \\
\hline J021902-082934 & 4.85 & $324 \pm 26$ & $228_{-13}^{+37}$ & $1.25_{-0.19}^{+0.29}$ & $1.85_{-0.21}^{+0.65}$ & $2.55_{-0.17}^{+0.13}$ & $0.71_{-0.06}^{+0.07}$ & $0.83_{-0.03}^{+0.13}$ \\
\hline J022056-063934 & 5.94 & $252 \pm 29$ & $256_{-12}^{+37}$ & $3.48_{-0.68}^{+0.89}$ & $2.81_{-0.25}^{+0.72}$ & $2.04+0.19$ & $0.34_{-0.14}^{+0.18}$ & $0.63_{-0.08}^{+0.19}$ \\
\hline J022511-045433 & 6.67 & $251 \pm 12$ & 287 & $2.82+0.59$ & 4.02 & $1.88_{-0.07}^{+0.07}$ & $0.55_{-0.08}^{+0.10}$ & $0.74+0.07$ \\
\hline J022610-042011 & 6.99 & $288 \pm 22$ & 279 & $2.74+0.55$ & 3.99 & $2.09+0.08$ & $0.21_{-0.15}^{+0.19}$ & $0.56_{-0.08}^{+0.08}$ \\
\hline J022648-090421 & 9.18 & $315 \pm 18$ & $315_{-23}^{+72}$ & $6.38^{+1.17}+1.27$ & $6.52_{-0.86}^{+2.41}$ & $2.16_{-0.09}^{+0.08}$ & $0.24_{-0.15}^{+0.16}$ & $\begin{array}{r}0.57+0.08 \\
-0.09\end{array}$ \\
\hline J023251-040823 & 5.46 & $282 \pm 18$ & $239_{-10}^{+31}$ & $1.64_{-0.27}^{+0.31}$ & $2.29_{-0.21}^{+0.74}$ & $2.27_{-0.12}^{+0.10}$ & $0.53_{-0.08}^{+0.10}$ & $0.73_{-0.05}^{+0.10}$ \\
\hline $\mathrm{J} 141137+565119$ & 4.32 & $246 \pm 21$ & 214 & $1.33_{-0.24}^{+0.29}$ & 1.45 & $2.30{ }_{-0.14}^{+0.12}$ & $0.45_{-0.10}^{+0.13}$ & $0.699_{-0.06}^{+0.12}$ \\
\hline $\mathrm{J} 221326-000946$ & 5.19 & $203 \pm 37$ & $241_{-13}^{+38}$ & $0.92_{-0.18}^{+0.20}$ & $2.17_{-0.21}^{+0.59}$ & $1.84_{-0.20}^{+0.19}$ & $0.12_{-0.23}^{+0.33}$ & $0.52_{-0.14}^{+0.19}$ \\
\hline $\mathrm{J} 221407-180712$ & 2.85 & $181 \pm 46$ & $171_{-9}^{+29}$ & $1.68_{-0.23}^{+0.32}$ & $0.57_{-0.06}^{+0.16}$ & $1.64_{-0.29}^{+0.53}$ & $0.03{ }_{-0.26}^{+0.35}$ & $0.39{ }_{-0.18}^{+0.53}$ \\
\hline $\mathrm{J} 221929-001743$ & 3.19 & $278 \pm 27$ & 197 & $2.44_{-0.40}^{+0.50}$ & 0.91 & $2.66_{-0.16}^{+0.13}$ & $0.02 \stackrel{+0.21}{-0.16}$ & $0.40_{-0.11}^{+0.13}$ \\
\hline
\end{tabular}

Note. - Only the 11 modeled lenses with measured velocity dispersions are shown. $\sigma_{\mathrm{e} 2}$ is the measured stellar velocity dispersion corrected to a standard aperture. $\sigma_{\mathrm{SIE}}$ values calculated from measured source redshifts are given without uncertainty and $\sigma_{\text {SIE }}$ values calculated from $z_{\text {s,pdf }}$ are given with the $16^{\text {th }}$ and $84^{\text {th }}$ percentiles (and were also weighted by the Sheth et al. fitting function and selection function). $M_{*}$ is the total stellar mass. $M_{\text {Ein }}$ is the total mass enclosed at the Einstein radius. Dark matter fractions are given at $R_{\text {eff }} / 2$ for both Salpeter and Chabrier IMFs. 